\title{
Picomolar Binding Selective Human Ron Inhibitory Antibodies Directed to Surface Displayed Conformational Epitopes for Imaging and Therapy
}

Xin Yu Koh

A*STAR: Agency for Science Technology and Research https://orcid.org/0000-0001-8323-9532

Xiao Hui Koh

A*STAR: Agency for Science Technology and Research

Diana Spiegelberg

Uppsala University: Uppsala Universitet

Preeti Jha

Uppsala University: Uppsala Universitet

Marika Nestor

Uppsala University: Uppsala Universitet

Le-ann Hwang

A*STAR: Agency for Science Technology and Research

Ban Xiong Tan

A*STAR: Agency for Science Technology and Research

David Lane ( $\sim$ dplane@p53lab.a-star.edu.sg )

P53 Laboratory, Agency for Science, Technology and Research (A*STAR) - Singapore, Singapore

\section{Research}

Keywords: Antibodies, receptor tyrosine kinase, RON, Narnatumab, immunoPET, radio-imaging

Posted Date: November 24th, 2020

DOI: https://doi.org/10.21203/rs.3.rs-111957/v1

License: (1) (1) This work is licensed under a Creative Commons Attribution 4.0 International License.

Read Full License 


\section{Abstract}

\section{Background}

The RON protein is an exceptionally interesting target in oncology and immunology. It is not only overexpressed in a wide variety of tumors but also has been shown to be expressed on myeloid cells associated with tumor infiltration, where it serves to dampen tumour immune response and reduce the efficacy of anti-CTLA4 therapy. Potent and selective inhibitory antibodies to RON might therefore both inhibit tumor cell growth and stimulate immune rejection of tumors.

\section{Methods}

We derived a new panel of exceptionally avid anti-RON antibodies with using traditional hybridoma technologies with picomolar binding affinities that inhibit MSP-induced RON signaling and show remarkable potency in antibody dependent cellular cytotoxicity (ADCC).

\section{Results}

When radiolabeled with 89-Zirconium, the new antibodies 3F8 and 10G1 also allow effective immunopositron emission tomography (immunoPET) imaging of RON-expressing tumors and recognize universally exposed RON epitopes at the cell surface.

\section{Conclusion}

In addition to their development as anti-RON cancer therapeutics, the new antibodies have great potential to be developed for tracking RON expressing tumours and metastases in cancer patients as diagnostic PET imaging agents, either as whole lgG or as a minibody.

\section{Background}

The RON receptor tyrosine kinase (MST1R) is expressed at high levels at the surface of many tumor cells of epithelial origin antibodies to RON have shown therapeutic potential in preclinical models both as intact antibodies [1, 2] and as antibody drug conjugates [3]. RON is a member of the MET receptor family and its sole ligand is the macrophage stimulating protein (MSP) [1]. Overexpressed RON is an oncogenic driver and small molecule inhibitors of the RON kinase and antibodies to the extracellular domain of RON have shown anti-tumor activity in a variety of pre-clinical models $[4,5]$. RON is expressed on myeloid cells and recent studies have shown that the anti-tumor activity of anti-CTLA4 antibodies is enhanced in RON knockout mice, suggesting that the inhibition of RON may enhance the activity of anti-CTLA4 treatment in enhancing host anti-tumor immunity [6]. Narnatumab, a humanized monoclonal against RON entered phase I clinical trials but failed for the lack of efficacy, in part because the antibody could not be given at high doses due to solubility issues [7]. 
We have previously reported a panel of mouse monoclonal antibodies to the unglycosylated extra cellular domain of RON and determined that they recognize cryptic epitopes which are present on intracellular forms of RON but are not exposed at the cell surface of cancer cells grown in tissue culture [2].

Surprisingly, we found one of the antibody, 6E6, which recognizes a sulfhydryl bond constrained epitope, to be highly effective in xenograft models despite the lack of apparent surface expression. Here we extend these studies by creating a second panel of antibodies directed against the glycosylated extra cellular domain of RON obtained from mammalian expression. The new antibodies show remarkable affinity for RON, determined using a kinetic exclusion assay, and bind tightly to the surface expressed form of the protein. Some antibodies in the panel act as antagonists blocking MSP signaling.

A key criterion in the development of antibody therapeutics for the treatment of cancer is to show preclinical evidence that the antibody therapeutic drug has remarkable anti-tumour efficacies with little toxicities. A novel image guided approach to monitor potential unspecific binding caused by the antibody drug would be through the use of companion diagnostics [8]. When labeled with a radiolabel, the antibody PET imaging agent can act as a tool to allow global tracking of the antigen expression in tumours or normal tissues in vivo, both as a predictor of therapeutic drug responses and any on-target off-tumour toxicities. In addition, in comparison to the traditional way of using immunohistochemistry staining of selected tissue biopsies as an indication of local tumour antigen expression, immunoPET companion diagnostic agents allow for a more complete and reliable view of antigen expression across the tumours in vivo, taking into considering any heterogeneity that might often be present in tumours [9]. Companion diagnostics can also be used as a predictor of the uptake of antibodies into tumour tissues or organs, as well as to detect possible differential antigen expression in metastases [10]. They are often used to provide valuable information on the pharmacokinetics and clearance of the antibodies in vivo [11]. From our panel of RON targeting antibodies, the antibodies with highest binding affinities- 10G1 with

a $2.9 \mathrm{pM} \mathrm{Kd}$ and $3 \mathrm{~F} 8$ with a $13.9 \mathrm{pM} \mathrm{Kd}$, were labelled with the radio-metal 89 -Zirconium $\left({ }^{89} \mathrm{Zr}\right)$ and showed excellent tumor specificity in PET imaging and developability as RON targeting radiopharmaceuticals.

\section{Methods}

\section{Generation of mouse monoclonal antibodies specific to mammalian extracellular RON}

ExpiCHO cells were used for expression of extracellular human RON protein from amino acids Gly25 to Thr957 fused to an N-terminal His-tag. The expressed protein was purified by Nickel affinity purification using fast purification liquid chromatography (FPLC) and used as an immunogen, following an optimized mouse immunization schedule [12]. Immunized mice were regularly checked for antibody titer against RON immunogen and a chosen mouse was sacrificed for hybridoma fusion. Cell supernatants from fused hybridoma cells were screened in ELISA against the immunogen and control His-tag protein for detection of specific binders. 
Breast cancer cell line T47D and colorectal cancer cell line HCT116 expressing endogenous RON were seeded in individual wells in a 96 well plate and allowed to adhere overnight. Cell culture supernatants from hybridoma cells were applied as primary antibodies for an hour following fixation by $4 \%$ paraformaldehyde. IgGs were detected with Alexa Fluor 488 conjugated anti-mouse IgG. Cells were counterstained with DAPI and viewed with the Incell Analyzer (GE Healthcare).

\section{Western blot analysis of RON antibody clones in cell lysates expressing endogenous and transfected RON}

Cells were harvested and lysed by sonication in $0.1 \%$ Triton X PBS supplemented with protease inhibitor cocktail (Roche). The QuickStart Bradford protein assay (BioRad) was used to determine protein concentration. BSA was used as a protein standard. $20 \mu \mathrm{g}$ of cell lysates were mixed with NuPAGE lithium dodecyl sulphate (LDS) and sample reducing buffer (Thermo Scientific), heated for 5 min at $95^{\circ} \mathrm{C}$ and loaded into 4-12\% Mini-PROTEAN precast gels (Biorad) for electrophoresis. Separated cell lysates were transferred onto nitrocellulose membranes using the Trans-Blot turbo transfer system device (Biorad). Blocking was performed with $5 \%$ milk or bovine serum albumin (BSA) in PBS supplemented with $0.1 \%$ tween (TBST). Hybridoma supernatants were applied to individual cell lysate strips as primary antibody and detected with goat anti-mouse IgG $(\mathrm{H}+\mathrm{L})$ (Jackson Laboratories). The enhanced chemiluminescence $(E C L)$ reagent used was SuperSignal West Dura Extended Duration Substrate (Thermo Scientific, \#34076). Imaging and acquisition were performed with Licor Odyssey Fc and Image Studio (Li-Cor Biosciences).

\section{Generation of RON knockout cell lines in HCT116, HT29 and T47D by CRISPR Cas9}

HCT116, HT29 and T47D cells were chosen for knockout of RONMST1R by CRISPR. A guide RNA containing the spacer (GGCGGGAGGAGCTCCATCG) that directs Cas9 to cut at the ATG initiation codon of MST1R was cloned into pX458, a plasmid, which contains the gRNA scaffold, spCas9-3xNLS and an EGFP reporter. This plasmid was transfected into HT29 and T47D cells using Lipofectamine 3000, and EGFP-positive cells were selected by FACS. Single clones were isolated and screened for MST1R knockout by directed Sanger sequencing near the Cas 9 cut site with the following primers (Forward: ggtccgctatcttggggc; Reverse: ctgggcaccacgtacttcac).

\section{Immunoprecipitation of RON with RON antibodies}

$1 X 10^{7}$ cells were harvested from T47D wildtype and T47D RON KO cell lines using RIPA buffer. $10 \mu \mathrm{g} / \mathrm{ml}$ of purified antibodies were added to $200 \mu \mathrm{g}$ of cell lysate and allowed to bind overnight at $4{ }^{\circ} \mathrm{C}$ in individual tubes. Antibody-RON complexes were picked up using Protein $\mathrm{G}$ Dynabeads magnetic beads (Thermo Scientific) and eluted in $20 \mathrm{mM}$ Tris-Glycine pH2.7 buffer. The eluted proteins were detected in western blotting using hydrogen peroxidase enzyme conjugated in-house anti-alpha RON antibody 6E6. SuperSignal West Dura Extended Duration Substrate (Thermo Scientific) was used for detection of chemiluminescence signal. Imaging and acquisition were performed using Licor Odyssey Fc and Image Studio (version 3.1). 
Flow cytometry analysis of RON antibodies with fixed and live cancer cells

$1 \mathrm{X} 10^{6}$ cells were harvested from T47D wildtype and T47D RON KO cell lines and washed in PBS. Cells were blocked and either paraformaldehyde fixed or stained live. Primary antibody staining was performed using $10 \mu \mathrm{g} / \mathrm{ml}$ of purified anti-RON antibodies. Cells were subsequently incubated with goat anti-mouse FITC-conjugated secondary antibodies (Invitrogen). FACS analysis was performed using FACS LSRII machine (Becton Dickinson). FlowJo (Tree Star Inc. USA) software was used for data analysis.

\section{Binding affinity studies of RON antibodies using Kinetic Exclusion Assay}

Mammalian produced recombinant extracellular His-tag RON protein was used for affinity measurements. Affinity determinations were carried out in the fixed antigen format. Antibodies were titrated as two-fold dilutions into a fixed concentration of RON antigen. RON protein was detected using mouse monoclonal to 6xHis-Tag (Dylight@650, Thermo Fisher). All affinity measurements were carried out using the KinExa 4000 (Sapidyne Instruments).

\section{Study of downstream RON antibodies signaling using phosphorylation assays}

60000 T47D cells and T47D RON ${ }^{-1}$ cells were seeded into individual wells in a 96 well plate. Varying concentrations of antibodies, wortmannin or control mouse sera were added to individual wells for an hour before addition of MSP $(10 \mathrm{nM})$ for half an hour. Experiments were run in triplicate. The cells were harvested for p-ERK level analyses using the AlphaLISA SureFire Ultra p-ERK1/2 (Thr202/Tyr204) Assay Kit (Perkin Elmer) as per manufacturer's protocol.

\section{Antibody dependent cellular cytotoxicity assay on RON antibodies}

All antibodies used for the antibody dependent cellular cytotoxicity assay were carried out with engineered chimeric RON antibodies which retained the mouse Fabs with a human Fc backbone and expressed recombinantly. Blood was collected from individuals that provided consent and all protocols were approved by the institutional review board (IRB). NK cells were isolated using the EasySep ${ }^{\text {TM }}$ Direct Human NK Isolation Kit (Stemcell Technologies). The effector to target ratio used was 10:1 based on target T47D cells initial seeding. ADCC activities were measured using the xCelligence platform (Roche Applied Science) using plates with detector electrodes that quantify the number of cells attached to the bottom of the wells, reflected by a calculated cell index $(\mathrm{Cl})$. The $\mathrm{Cl}$ was measured every 15 minutes over 72 hours after the antibody treatment. Treatments were performed in triplicates, with averages and standard deviations calculated by the instrument.

\section{In vivo animal imaging studies on 3F8 and 10G1}

In vivo xenograft model

Female nu/nu Balb/c mice $(n=8)$ were housed under standard laboratory conditions and fed ad libitum. All experiments complied with Swedish law and were performed with permission from the Uppsala 
Committee of Animal Research Ethics. Tumor xenografts were formed by subcutaneous inoculation of approximately $1 \times 10^{6}$ RON-positive HT29 cells on the right posterior leg and $1 \times 10^{6}$ RON null HCT116 cells on the left posterior leg.

DFO-conjugation and ${ }^{89}$ ZrRadiolabeling of $10 G 1$ and $3 F 8$

Conjugation of p-SCN-Bn-Deferoxamine (DFO) to Ab's and ${ }^{89} \mathrm{Zr}$-labelling was performed as described previously [13]. In brief, Ab's (2mg/ml dissolved in $0.07 \mathrm{M}$ borax buffer, $\mathrm{pH} 9.4)$ were incubated with the bifunctional chelator DFO (B-705, Macrocyclics Dallas, TX, USA) in the molar ratio of 1:3 (antibody to DFO) for $1 \mathrm{~h}$ at $37^{\circ} \mathrm{C}$ using a thermomixer at $350 \mathrm{rpm}$. Unbound-DFO and Ab-DFO were separated with a NAP-5 column equilibrated with $0.25 \mathrm{M}$ ammonium acetate ( $\mathrm{pH} 5.4-5.6)$.

$20 \mathrm{MBq}{ }^{89} \mathrm{Zr}$-oxalic (solid target production, clinical grade; kindly provided by $\mathrm{Dr}$. Thuy Tran, $\mathrm{KI}$, Stockholm) acid solution was added to $400 \mu \mathrm{g} \mathrm{Ab}$ at pH 6.8-7.2 (0.1 $\mathrm{M} \mathrm{Na}_{2} \mathrm{CO}_{3}$ and 0.5 M HEPES were added for $\mathrm{pH}$ adjustment) and incubated for $1 \mathrm{~h}$ at room temperature while gently shaking at $350 \mathrm{rpm}$. Radiolabeling efficiency and radionuclidic purity (typically $>96 \%$ ) was determined by chromatography strips (ITLC) using $0.2 \mathrm{M}$ citric acid ( $\mathrm{pH}$ 4.9-5.1) as mobile phase and analysis using a Fujifilm Bas-1800II phosphorimager (Fuji, Tokyo, Japan).

In vitro radioimmunoassay

$1 \mathrm{nM}$ of ${ }^{89} \mathrm{Zr}-10 \mathrm{G} 1$ or ${ }^{89} \mathrm{Zr}-3 \mathrm{~F} 8$ was added to approximately $0.5^{\star} 10^{6} \mathrm{HT} 29$ (RON +) or HCT116 (RON KO) cells, and incubated at $37^{\circ} \mathrm{C}, 5 \% \mathrm{CO}_{2}$. After $24 \mathrm{~h}$, cells were washed, trypsinized and counted. Cellassociated radioactivity was measured in a gamma counter (1480 Wizard 3", Wallace, Turku, Finland). Radioactivity count was adjusted for cell number, and the signal on HT29 cells was normalized to HCT116 signal using GraphPad Prism 8 (GraphPad Software, San Diego, CA, USA). Statistical analysis of differences in uptake between antigen-positive and antigen-negative cells was performed using Graph Pad Prism 8, using unpaired student's t-test, with $p<0.05(*), p<0.01(* \star)$, and $p<0.001(* \star *)$.

PET imaging of ${ }^{89} \mathrm{Zr}-10 \mathrm{G} 1$ and ${ }^{89} \mathrm{Zr}-3 \mathrm{~F} 8$

Xenografted mice were injected via the tail vein with $50 \mu \mathrm{g}$ of ${ }^{89} \mathrm{Zr}-10 \mathrm{G} 1 \quad(\mathrm{n}=4)$ or ${ }^{89} \mathrm{Zr}-3 \mathrm{~F} 8(\mathrm{n}=4)$ antibodies (injected activity 1.1 and $0.8 \mathrm{MBq}$ ), respectively. Whole-body PET/MRI/CT studies were performed under general anesthesia (sevoflurane $2.0-3.5 \%$ in $50 \% / 50 \%$ medical oxygen + air at $60 \mathrm{ml} /$ $\min$ ) after $24 \mathrm{~h}, 48 \mathrm{~h}$ and 72 p.i. (i.v.) for ${ }^{89} \mathrm{Zr}-10 \mathrm{G} 1(\mathrm{n}=2)$ and ${ }^{8} 9 \mathrm{Zr}-3 \mathrm{~F} 8(\mathrm{n}=2)$. Pre-injected mice were placed under sedation in the gantry of a small-animal nanoScan PET/MR scanner (Mediso Medical Imaging Systems Ltd., Hungary) and a whole-body PET scan was performed for 60 min in list mode followed by a CT scan in nanoScan SPECT/CT scanner (Mediso Medical Imaging Systems Ltd., Hungary) for $5 \mathrm{~min}$. The breathing rate was monitored and animals were placed on the heated bed to prevent hypothermia. PET data was reconstructed into a static image and corrected for the time of injection using the Tera-Tomo ${ }^{\mathrm{TM}} 3 \mathrm{D}$ reconstruction (6 subsets and 4 iterations). The raw $\mathrm{CT}$ data was reconstructed 
using filtered back projection. PET and CT Dicom files were analyzed with PMOD v3.510 (PMOD Technologies Ltd, Zurich, Switzerland).

Biodistribution of ${ }^{89}$ Zr-10G1 and ${ }^{89}$ Zr-3F8

Biodistribution of RON antibodies in xenografts was studied 72 hours p.i. following PET analyses for

${ }^{89} \mathrm{Zr}-10 \mathrm{G} 1(\mathrm{n}=4)$ and ${ }^{89} \mathrm{Zr}-3 \mathrm{~F} 8(\mathrm{n}=4)$. Animals were euthanized with a mixture of ketamine and xylazine followed by heart puncture. Blood was collected, HCT116 tumors, thyroid (en bloc with larynx), heart, liver, kidneys, spleen, urinary bladder, colon, upper gastrointestinal tract, skin, bone and muscle were excised, weighed and measured in a gamma well-counter (1480 Wizard; Wallace Oy, Turku, Finland). Injection standards were measured for each time point. Radioactivity uptake in the organ was calculated as the percentage of injected dose per gram of tissue (\%ID/g). Thyroid uptake was calculated as the percentage of injected dose per organ (\%ID/organ).

Statistical analysis of differences in uptake between antigen-positive and antigen-negative tumors was performed with Graph Pad Prism 8 (GraphPad Software, San Diego, USA), using unpaired student's t-test, with $\left.p<0.05(*), p<0.01{ }^{(* *}\right), p<0.001\left(^{(* \star}\right)$ and $p<0.0001(* \star \star *)$.

\section{Results}

\section{Screening and characterization of antibodies targeting membrane bound RON}

The unusual behavior of the antibodies that we produced when we immunized mice with bacterially produced RON protein domains has been described previously [2]. Essentially, we found antibodies that worked exceptionally well in immunoblotting and immunohistochemistry applications but showed weak surface binding to live cells in flow cytometry. Treatment of cells with mild fixatives or inhibitors of glycosylation massively enhanced specific binding in flow cytometry. Despite the apparent cryptic nature of the epitopes recognized by these antibodies, several of them showed activity in xenograft assays.

In an effort to understand these results in more detail, we compared the activity of the antibodies in ELISA and SPR assays directed towards our original immunogens and preparations of the ECD of RON expressed in, and purified from, mammalian cells. This protein extended from amino acid Gly 25 to Thr 957 and contained an $\mathrm{N}$ terminal His-tag. Surprisingly, the antibodies only bound to these RON preparations weakly, if at all in either SPR or ELISA formats (Data not shown). To confirm that the correct protein had been purified the preparation was analyzed by protease digestion and mass spectrometry (MS). The results showed that the protein was of the correct sequence but peptide containing the N-X-S/T motif required for glycosylation were undetectable by MS. This suggested that the preparation was highly glycosylated. It was however bound by a polyclonal antibody to RON, suggesting that it displayed novel epitopes not detected by our existing panel of antibodies. We therefore set out to make a new panel of anti-RON monoclonal antibodies using the mammalian produced His-tagged RON preparation as the immunogen. In order to select for novel antibodies, we used the immunogen in an ELISA assay to screen the mice after immunization and to screen the hybridoma fusion, and supplemented this screening assay 
with the immunofluorescent staining of two human cancer cell lines that express RON (HCT116 and T47D) in a high throughput 96 well based assay using Incell Analyzer (GE Healthcare). The unusual behavior of our first set of antibodies in failing to bind to the mammalian RON preparation motivated us to use CRISPR Cas9 to "knock-out" the RON gene from three cell lines (HCT116, HT29 and T47D cells) to use as controls in counter screening assays.

Mice were immunized with the mammalian-produced RON protein in adjuvant, and mouse sera from one of the animals showed a high titre in the ELISA assay (Figure $1 \mathrm{~A}$ ). In this assay we compared binding to the His-tagged RON protein to binding to another His-tagged protein. While the commercial anti-RON antibody and the mouse serum from our immunized donor mouse showed binding to both proteins, as did a commercial antibody to the His-tag, the eight selected new mouse monoclonal antibodies were shown to bind strongly to the mammalian cell produced His-tagged RON but not to the control His-tagged protein.

We next examined the binding of the antibodies to the cell surface of live unfixed human cancer cell lines T47D and HCT116. To block receptor mediated endocytosis, we fixed the cells with $4 \%$ paraformaldehyde after adding primary antibodies and washing off unbound antibodies before detecting bound antibodies with an Alex Fluor conjugated anti-mouse IgG antibody. All of the eight monoclonal antibodies showed strong cell surface staining by confocal microscopy, while a mouse IgG control gave only a weak background stain (Figure 1B). As an additional control we used the CRISPR RON knockout cells in the same protocol and showed that none of the new antibodies could stain these cells, providing strong evidence that the antibodies were RON specific and devoid of non-specific cell surface binding. We next tested the antibodies for their ability to immunoprecipitate the RON protein from cell extracts. To probe the blot, we used the anti-RON alpha chain monoclonal antibody $6 \mathrm{E} 6$ directly conjugated to peroxidase. This method gave extremely clean results. The control anti-RON antibody, Narnatumab, immunoprecipitated both the free alpha chain of RON at $35 \mathrm{Kda}$ and uncleaved pro-RON at $150 \mathrm{KDa}$ respectively. As expected, nothing was detected in the RON knock-out cell immune precipitates. Our new monoclonal antibodies showed strong and specific immunoprecipitation of the 150kDa pro-RON and the $35 \mathrm{kDa}$ RON alpha chain species, like Narnatumab.

Finally, we tested the antibodies' ability to bind to cell surface RON using flow cytometry. In our prior study we had found that the antibody $6 \mathrm{E} 6$ only bound to the cell surface after mild fixation with paraformaldehyde but could not bind to unfixed live cells. We therefore compared binding of the new panel of antibodies to both live cells and PFA fixed cells. In the live cell assay Narnatumab and seven of the eight new antibodies showed a clear completely right shifted population of strongly stained T47D cells (lighter peak) but no staining in RON knockout T47D cells (lighter peak) (Figure 1D). No staining was observed with the new antibody 9F6 or with any of the isotype control or secondary only antibodies.

Similarly, Narnatumab and all the new antibodies stained PFA-fixed cells, with the exception of 9F6. None of the antibodies stained the isogenic T47D RON KO cells, suggesting that the antibodies were highly specific to RON. 


\section{Determination of the binding affinities of the antibodies by KinEXa}

An initial analysis of the binding of the new antibodies using Surface Plasmon Resonance revealed that many of the new antibodies had exceptionally slow off rates making a true determination of their Kds problematic. This limitation of SPR for highly avid antibodies has been described previously and the use of kinetic exclusion assay has been proposed as a way to overcome this limitation allowing measurements in the sub nanomolar range. We employed the Kinetic Exclusion assay (KinEXa) method to measure the $\mathrm{Kd}$ values of all of the new antibodies using a fixed concentration of the recombinant mammalian expressed protein as the target antigen and a twelve point dilution curve of each antibody. We dropped antibody $9 \mathrm{~F} 6$ in this assay as it seemed less avid than the other antibodies in preliminary SPR binding assays. The remaining seven antibodies gave reproducible results in duplicate analysis (Figure 2). The binding data and the resultant Kd values showed a wide range of affinities, with four (3F8, $3 G 4,7 H 11$ and 10G1) of the seven antibodies binding with sub-nanomolar affinities. The $10 \mathrm{G} 1$ antibody stood out with the exceptionally high binding affinity of 29 pM.

\section{Extracellular RON antibodies blocks MSP induced downstream signaling pathways}

Next, we sought to determine if our new panel of antibodies to RON could inhibit the activation of the RON signaling pathway by MSP. First, we analyzed a panel of cell lines and studied the response to MSP by using immunoblotting for phosphorylated ERK 2 (Thr202/Tyr204) (Figure 3A). While several of the cell lines responded to MSP stimulation with increased levels of phosphorylated ERK, the clearest signal was seen with the T47D cells line. Other cell lines showed weaker responses (HCT116 cells) or had high levels of ligand independent pERK (MDA MB231 cells). AlphaScreen was subsequently used to quantitatively measure the antibody-mediated inhibition of RON signaling upon MSP stimulation. While two of the antibodies (3F6 and 9F6) were not antagonistic (Figure 3B), the remaining six antibodies showed dosedependent inhibition of RON signalling (Figure 3C), with IC50s ranging from $2.8 \mathrm{nM}$ of $10 \mathrm{G} 1$ to $68 \mathrm{nM}$ for 3G4 (Figure 3D). In comparison, Narnatumab had an $\mathrm{IC}_{50}$ of $14.9 \mathrm{nM}$, an order of magnitude less potent than 10G1 (Figure 3E).

In our screening studies, western blot was performed on full length RON transfected 293FT cells or HCT116 cells expressing endogenous RON with our hybridoma supernatants as primary antibodies. However, none of the eight antibodies, unlike 6E6, were able to detect the RON bands on either RON transfected cell lysates or lysates expressing endogenous RON, implying that the antibodies are unable to detect the denatured form of RON produced in the immunoblotting method and detect only folded RON protein in applications like cell staining, ADCC or flow cytometry analyses. (Table 1) This suggests that the epitope the antibodies bind to is lost upon denaturation.

\section{RON antibodies exhibit therapeutic efficacy through ADCC}

One key mechanism by which therapeutic monoclonal antibodies can exert their effects is through the Fc dependent recruitment of immune effector cells that mediate antibody dependent cell cytotoxicity (ADCC). For this assay we used purified human NK cells and titrated the eight new antibodies over a wide 
range of concentrations (from $0.1 \times 10^{-12} \mathrm{~g} / \mathrm{ml}$ to $1 \times 10^{-6} \mathrm{~g} / \mathrm{ml}$ ) using an impedance-based cell cytotoxicity assay (xCELLigence). The assay allows real time monitoring of target cell lysis after the addition of antibodies and effector cells. The plots show (Figure 4B) that five of the antibodies showed ADCC activity, with dose dependent cell killing detectable at the first time point (40 minutes) but varied greatly in their potency. Specific lysis data (Figure 4A and 4B) were calculated from normalized cell index and baseline (no effector cells) subtracted curves using the xCELLigence RTCA data analysis software. We compared the antibodies to the clinically important anti-HER2 antibody Trastuzumab in this assay and found in keeping with literature that it had an $\mathrm{EC}_{50}$ of around $13 \mathrm{pM}$. Two of our antibodies (3F8 and 3G4) showed similar values to Trastuzumab while again the $10 \mathrm{G} 1$ antibody showed exceptional activity with an $\mathrm{EC}_{50}$ value of $3.0 \mathrm{pM}$.

\section{Radiolabeled RON antibodies are specifically localized in RON expressing tumours}

The use of radiolabeled antibodies both for imaging as well as therapeutic radiopharmaceuticals is gaining increased interest. We labeled the 10G1 and 3F8 antibody directed to a surface exposed epitope with ${ }^{89} \mathrm{Zr}$ after DFO-conjugation. Both ${ }^{89} \mathrm{Zr}$-labeled $10 \mathrm{G} 1$ and $3 \mathrm{~F} 8$ antibodies displayed a significantly higher signal on RON positive (HT29) cells compared to RON negative (RON null HCT116) cells in vitro (Figure 5E).

For the in vivo imaging study, we prepared mice with two xenografts, one on each flank. The tumour on the right flank was formed from the RON wild type protein expressing cell HT29, while the tumour on the left flank was formed of RON knockout HCT116 cells. PET imaging of the mice at 24,48 and $72 \mathrm{~h}$ post injection of the ${ }^{89} \mathrm{Zr}$-labeled antibodies, as well as biodistribution studies ex vivo at $72 \mathrm{~h}$ post injection, showed selective accumulation of the ${ }^{89} \mathrm{Zr}$-labeled antibodies in the RON positive tumors on the right flank (Figure 5), significantly higher than for the RON-negative tumors on the left flank. Biodistribution studies confirmed the specific uptake in the RON positive tumors, as well as high signals in the blood and blood-rich organs like liver and spleen, probably due to the long circulation time of the antibodies (Figure $5 \mathrm{D})$.

\section{Discussion}

\section{A new panel of antibodies to RON}

While a vast array of recombinant methods has been devised to produce therapeutic antibodies, these are generally complex and expensive. The use of huge phage libraries has allowed the production of clinically remarkably successful antibodies such as Humira while the use of humanized mice has also been successful and produced antibodies like FDA approved anti-EGFR antibodies Cetuximab and Panitumumab [14, 15]. More recently, B cell cloning using antigen selections has become effective [16]. However, despite these advances the traditional methods of hybridoma production are very well established and can take advantage of decades of experience in immunization protocols and screening methods [17]. Indeed, many of the best-selling therapeutic antibodies have been developed with this 
workflow, using recombinant methods to humanize the initial mouse antibodies and improve their biophysical properties [18]. Most recently the use of antibody drug conjugates, bi-specific antibodies, and recycling antibodies, along with the development of the CAR-T cells has enormously enhanced the potential of developing tumour specific immunotherapies $[19,20]$.

Receptor tyrosine kinases overexpressed and mutated in cancer cells have proven to be exceptionally good targets for such approaches and antibodies to the EGFR and HER2 receptors have proved very useful in the treatment of breast cancer and colon cancer [21]. However, to date, no successful clinical antibodies to the related Met and RON receptors have been produced [1]. The reasons for failure can be many and the tested molecules were only tried as simple antibodies, instead of modalities like drug conjugates or recycling antibodies. As such, their efficacies depend solely on signaling blockade, induction of the complement cascade, or Fc-mediated recruitment of NK cells or macrophages [22]. Here we generate a panel of new antibodies to RON and show superior binding and biological activity to Narnatumab, overcoming the problems faced in the latter's clinical development. In particular, the 10G1 antibody binds with picomolar affinity (Kd) and is active in ADCC assays at single digit picomolar concentrations. 10G1 also performs exceptionally well as an in vivo imaging agent and is a suitable candidate for further development and antibody engineering.

\section{RON antibodies display profiles suitable for clinical development as cancer therapeutics}

The cost of bringing an antibody molecule from 'bench to bedside' is remarkable. A study done from collecting data from 13 big pharmaceuticals estimated the cost of development of one new biologic molecule to be around $\$ 1.8$ billion USD [23]. As such, it is critical that most effort should be put into the selection of lead antibody candidates at the early discovery stage, to ensure the highest success rate for the antibody to reach the clinic [24]. Despite the initial promising biological functions shown by RON targeting antibody Narnatumab in preclinical models, the failure of the molecule when tested in phase I clinical trials was partially attributed to the molecule's poor biophysical properties [7]. Lead monoclonal antibody candidates for drug development are typically selected for their high affinities and specificities to the target, potency and biological activities, and abilities to evoke Fc receptor functions [25]. From our panel of new anti-RON antibodies, our antibody with the highest binding affinity of 2.9 pM $10 \mathrm{G} 1$ was chosen as the lead candidate for its ability to specifically bind to and immunoprecipitate RON expressed on the surface of cancer cells, potently block MSP stimulated downstream signaling of RON receptor, and its ability to elicit strong antibody dependent cellular cytotoxicity (ADCC) responses which can aid in tumour cell elimination. Moreover, the antibody can be recombinantly expressed and purified at a higher yield than Narnatumab, and is stable and soluble at high concentrations, without the biophysical

problems of Narnatumab. Even though there is a need for 10G1 to go through more rigorous testing of its biophysical properties to ensure its developability, $10 \mathrm{G} 1$ appears to be a promising lead candidate at this stage of development.

\section{Zirconium radiolabeled-10G1 can be developed into companion diagnostics for clinical imaging of RON positive tumours}

Page 11/33 
The use of radiolabeled antibodies for the non-invasive diagnostic and detection of tumours biomarkers on primary tumours and metastases across different tumour types has been long established since the 1980s [26]. Our preliminary study using ${ }^{89} \mathrm{Zr}-10 \mathrm{G} 1$ and ${ }^{89} \mathrm{Zr}$-3F8 showed that the antibodies were able to specifically colocalize and accumulate in the xenograft tumour expressing RON with little cross reactivity to the RON knockout tumour. Whereas the antibodies demonstrated similar ratios for RON positive vs RON knockout tumors, ${ }^{89} \mathrm{Zr}-10 \mathrm{G} 1$ demonstrated higher tumour uptake and a slower excretion from blood compared to ${ }^{89} \mathrm{Zr}-3 \mathrm{~F} 8$. Even though our study demonstrated promising data with the antibodies as in a whole IgG format, a potential improvement could be to explore their use as immunoPET agents as smaller antibody fragments like a minibody, single-chain variable fragment (scFv) or antigenbinding fragment (Fab), specifically for a few reasons. Firstly, murine based antibodies have a risk of inducing human anti-mouse antibody (HAMA) activity in human patients, using a smaller antibody fragment will reduce the likelihood of this incident [27]. Also, antibody fragments that are smaller in size may have better tissue penetration [28]. Thirdly, the antibodies will have a shorter circulation time in blood, allowing for a higher tumour to blood ratio and a shorter duration for maximum contrast imaging [29]. As clinical research in oncology moves towards precision medicine, the development of immunoPET agents like 10G1 as companion diagnostics is critical to aid in the achievement of this goal, allowing for an improvement in the method for diagnosing RON related malignancies in the clinic.

\section{Conclusion}

Our studies on antibodies to RON have a general significance in antibody development to the extracellular domain of tyrosine kinase receptors for diagnosis, therapy and imaging. Perhaps, the most striking observation has been the profound effect the immunogen has on the types of antibodies produced. The bacterially produced antigen [2] and the mammalian cell produced antigen have both induced useful and highly specific antibodies. The antibodies to the bacterial produced antigen work very well in immunoprecipitation, immunohistochemistry and in immunoblotting. Their epitopes can be precisely mapped using synthetic peptide libraries or phage peptide libraries. They react strongly to intracellular forms of RON and can be used in flow cytometry of fixed cells. Their reaction to the RON protein expressed at the surface of cancer cells is weak however and is induced by treatment with tunicamycin [2]. The new antibodies described here show that this weak surface staining is not a feature of all antibodies to RON as the new antibodies in response to the mammalian protein immunogen this new panel of antibodies show strong cell surface binding and are highly effective in inhibiting MSP driven signalling and in the activation of ADCC with human donor NK cells. The epitopes of the new antibodies could not be mapped by phage peptide libraries or synthetic peptide libraries and the antibodies are unable to detect antigen by immunoblotting being directed to conformational epitopes. The immunogen thus defines the dominant epitopes recognised. While glycosylation of the mammalian produced RON protein is probably a major factor in this selection the panel of antibodies reveals unexpected features of RON expression in cancer cells as antibodies to the bacterially produced protein proved highly active in xenograft models. These two complementing panels of antibodies will permit a more thorough understanding of RON epitope exposure in human cancers through their use a imaging agents and in

Page 12/33 
proteomic analysis of intracellular and extracellular forms of the RON protein. As has been the case with the EGFR protein and its therapeutic antibodies targeting different epitopes [30], it may thus be possible to develop antibody therapies that are directed against cancer cell expressed or immune suppressor cell expressed forms of RON, extending the value of RON as a target in the immunotherapy of cancer and permitting image guided personalised medicine.

\section{List Of Abbreviations}

ADCC: antibody dependent cellular cytotoxicity; immunoPET: immuno-positron emission tomography; RON: receptor de'origin Nantais; CTLA-4: cytotoxic T-lymphocyte-associated protein 4; MSP: macrophage stimulating protein; FPLC: fast purification liquid chromatography; ELISA: enzyme-linked immunosorbent assay; LDS: lithium dodecyl sulphate; BSA: bovine serum albumin; ECL: enhanced chemiluminescence; EGFP: enhanced green fluorescent protein; FACS: fluorescence-activated cell sorting; $p$-ERK: phosphorextracellular-signal-regulated kinase; KinExa: kinetic exclusion assay; NK: natural killer; DFO: deferoxamine; KO: knock-out; PET/MR: positron emission tomography-magnetic resonance; CT: computed tomography; SPECT: single-photon emission computerized tomography; PET: positron emission tomography; SPR: surface plasmon resonance; MS: mass spectrometry; CRISPR: clustered regularly interspaced short palindromic repeats; HER2: human epidermal growth factor receptor 2; HAMA: human anti-mouse antibody; EGFR: epidermal growth factor receptor; scFv: single-chain variable fragment; Fab: antigen binding fragment

\section{Declarations}

\section{Ethics approval and consent to participate}

All experiments complied with Swedish law and were performed with permission from the Uppsala Committee of Animal Research Ethics

Consent for publication

Not applicable

\section{Availability of data and material}

Datasets used and analyzed for this study can be provided from the corresponding author upon reasonable request

\section{Competing interests}

Authors declare no potential conflicts of interest 


\section{Funding}

This study was supported by funding from A*STAR (Agency of Science, Technology and Research). The preclinical molecular imaging work in this publication was performed at Preclinical PET-MRI Platform, Department of Medicinal Chemistry, Uppsala University, Sweden. The Platform is supported by the Knut and Alice Wallenberg Foundation, Science for Life Laboratory and Disciplinary domain of medicine and pharmacy, Uppsala University. This study was supported by grants from the Swedish Cancer Society (CAN 2018/494, CAN 20 0191).

\section{Authors' contributions}

DPL, HLA, MN and KXY contributed to the conception and design of the study. HLA, MN and DPL supervised the study. $\mathrm{KXH}, \mathrm{KXY}, \mathrm{DS}$ and PJ contributed to the acquisition of data. DPL, KXY, KXH, MN and DS took part in the analysis and interpretation of data. KXY, DPL, TBX and MN took part in the writing, review and/ or revision of the manuscript. All authors read and approved the manuscript.

\section{Acknowledgements}

The authors would like to thank Dr. Khian Hong Pua for critical reading of the manuscript.

\section{References}

1. Yao, H.P., et al., MSP-RON signalling in cancer: pathogenesis and therapeutic potential. Nat Rev Cancer, 2013. 13(7): p. 466-81.

2. Koh, X.Y., et al., Therapeutic anti-cancer activity of antibodies targeting sulfhydryl bond constrained epitopes on unglycosylated RON receptor tyrosine kinase. Oncogene, 2019. 38(48): p. 7342-7356.

3. Tong, X.-M., et al., Therapeutic efficacy of a novel humanized antibody-drug conjugate recognizing plexin-semaphorin-integrin domain in the RON receptor for targeted cancer therapy. Journal for ImmunoTherapy of Cancer, 2019. 7(1): p. 250.

4. Zou, Y., et al., Ron knockdown and Ron monoclonal antibody IMC-RON8 sensitize pancreatic cancer to histone deacetylase inhibitors (HDACi). PLoS One, 2013. 8(7): p. e69992.

5. Yao, H.-P., et al., The monoclonal antibody $Z t / f 2$ targeting $R O N$ receptor tyrosine kinase as potential therapeutics against tumor growth-mediated by colon cancer cells. Molecular cancer, 2011. 10: p. 8282.

6. Ekiz, H.A., et al., Inhibition of RON kinase potentiates anti-CTLA-4 immunotherapy to shrink breast tumors and prevent metastatic outgrowth. Oncoimmunology, 2018. 7(9): p. e1480286-e1480286.

7. LoRusso, P.M., et al., Phase 1 study of narnatumab, an anti-RON receptor monoclonal antibody, in patients with advanced solid tumors. Invest New Drugs, 2017. 35(4): p. 442-450.

8. Carmon, K.S. and A. Azhdarinia, Application of Immuno-PET in Antibody-Drug Conjugate Development. Molecular imaging, 2018. 17: p. $1536012118801223-1536012118801223$. 
9. McKnight, B.N. and N.T. Viola-Villegas, (89) Zr-ImmunoPET companion diagnostics and their impact in clinical drug development. Journal of labelled compounds \& radiopharmaceuticals, 2018. 61(9): p. 727-738.

10. Fischer, M. and W.U. Kampen, Radionuclide Therapy of Bone Metastases. Breast care (Basel, Switzerland), 2012. 7(2): p. 100-107.

11. Matthews, P.M., et al., Positron emission tomography molecular imaging for drug development. British journal of clinical pharmacology, 2012. 73(2): p. 175-186.

12. Gustafsson, B., Immunizing schedules for hybridoma production. Methods Mol Biol, 1990. 5: p. 5979.

13. Vosjan, M.J., et al., Conjugation and radiolabeling of monoclonal antibodies with zirconium-89 for PET imaging using the bifunctional chelate p-isothiocyanatobenzyl-desferrioxamine. Nat Protoc, 2010. 5(4): p. 739-43.

14. Nelson, A.L., E. Dhimolea, and J.M. Reichert, Development trends for human monoclonal antibody therapeutics. Nature Reviews Drug Discovery, 2010. 9(10): p. 767-774.

15. Lu, R.-M., et al., Development of therapeutic antibodies for the treatment of diseases. Journal of Biomedical Science, 2020. 27(1): p. 1.

16. DeKosky, B.J., et al., High-throughput sequencing of the paired human immunoglobulin heavy and light chain repertoire. Nature Biotechnology, 2013. 31(2): p. 166-169.

17. Deckert, P.M., Current Constructs and Targets in Clinical Development for Antibody-Based Cancer Therapy. Current Drug Targets, 2009. 10(2): p. 158-175.

18. Chiu, M.L., et al., Antibody Structure and Function: The Basis for Engineering Therapeutics. Antibodies, 2019. 8(4): p. 80.

19. Igawa, T., et al., Antibody recycling by engineered $\mathrm{pH}$-dependent antigen binding improves the duration of antigen neutralization. Nature Biotechnology, 2010. 28(11): p. 1203-U96.

20. Goulet, D.R. and W.M. Atkins, Considerations for the Design of Antibody-Based Therapeutics. Journal of Pharmaceutical Sciences, 2020. 109(1): p. 74-103.

21. Maroun, C.R. and T. Rowlands, The Met receptor tyrosine kinase: A key player in oncogenesis and drug resistance. Pharmacology \& Therapeutics, 2014. 142(3): p. 316-338.

22. Graziano, R.F. and J.J. Engelhardt, Role of Fc gamma Rs in Antibody-Based Cancer Therapy. Fc Mediated Activity of Antibodies: Structural and Functional Diversity, 2019. 423: p. 13-34.

23. Paul, S.M., et al., How to improve R\&D productivity: the pharmaceutical industry's grand challenge. Nature Reviews Drug Discovery, 2010. 9(3): p. 203-214.

24. Mellman, I., et al., De-Risking Immunotherapy: Report of a Consensus Workshop of the Cancer Immunotherapy Consortium of the Cancer Research Institute. Cancer Immunology Research, 2016. 4(4): p. 279-288.

25. Bailly, M., et al., Predicting Antibody Developability Profiles Through Early Stage Discovery Screening. Mabs, 2020. 12(1): p. 28. 
26. Hong, H., J. Sun, and W. Cai, Radionuclide-Based Cancer Imaging Targeting the Carcinoembryonic Antigen. Biomarker insights, 2008. 3: p. 435-451.

27. Tjandra, J.J., L. Ramadi, and I.F. McKenzie, Development of human anti-murine antibody (HAMA) response in patients. Immunol Cell Biol, 1990. 68 (Pt 6): p. 367-76.

28. Kaur, S., et al., Recent trends in antibody-based oncologic imaging. Cancer letters, 2012. 315(2): p. 97-111.

29. Fu, R., et al., Antibody Fragment and Affibody ImmunoPET Imaging Agents: Radiolabelling Strategies and Applications. ChemMedChem, 2018. 13(23): p. 2466-2478.

30. Martinelli, E., et al., Anti-epidermal growth factor receptor monoclonal antibodies in cancer therapy. Clinical and experimental immunology, 2009. 158(1): p. 1-9.

\section{Tables}

Table 1 Summary table showing the characterization of anti-RON antibodies by binding assays 


\section{Table 1}

\begin{tabular}{|c|c|c|c|c|c|c|c|c|}
\hline $\begin{array}{l}\text { Clone } \\
\text { name } \\
\text { Assay } \\
\text { performed }\end{array}$ & $3 G 4$ & $3 \mathrm{~F} 6$ & $3 F 8$ & $6 A 9$ & $7 \mathrm{H} 11$ & 9F2 & $9 \mathrm{~F} 6$ & 10G1 \\
\hline ELISA & +++ & +++ & +++ & +++ & +++ & +++ & ++ & +++ \\
\hline $\begin{array}{l}\text { WB- } \\
\text { Trans }\end{array}$ & - & - & - & - & - & - & - & - \\
\hline WB- Endo & - & - & - & - & - & - & - & - \\
\hline $\begin{array}{l}\text { Cell } \\
\text { staining- } \\
\text { Trans }\end{array}$ & +++ & +++ & +++ & +++ & +++ & +++ & ++ & +++ \\
\hline $\begin{array}{l}\text { Cell } \\
\text { staining- } \\
\text { Endo }\end{array}$ & $\begin{array}{l}+++ \\
(\mathrm{Me} \\
\mathrm{m})\end{array}$ & $\begin{array}{l}+++ \\
(\mathrm{Mem})\end{array}$ & $\begin{array}{l}+++ \\
\text { (Mem) }\end{array}$ & $\begin{array}{l}+++ \\
\text { (Mem) }\end{array}$ & $\begin{array}{l}+++ \\
\text { (Mem) }\end{array}$ & $\begin{array}{l}+++ \\
(\mathrm{Mem})\end{array}$ & $\begin{array}{l}+++ \\
\text { (Mem) }\end{array}$ & $\begin{array}{l}+++ \\
\text { (Mem) }\end{array}$ \\
\hline $\begin{array}{l}\text { Cell } \\
\text { staining- } \\
\text { RON KO }\end{array}$ & - & - & - & - & - & - & - & - \\
\hline $\begin{array}{l}\text { FACS } \\
\text { (T47D } \\
\text { cells) } \\
\end{array}$ & ++ & ++ & ++ & ++ & ++ & ++ & ++ & ++ \\
\hline IP & +++ & +++ & +++ & +++ & +++ & +++ & +++ & +++ \\
\hline
\end{tabular}

* Trans stands for transfected cells, Endo stands for endogenous RON expressing cells, RON KO stands for RON knock-out cell HCT116 cells. Mem indicates membrane staining. IP stands for immunoprecipitation

\section{Figures}




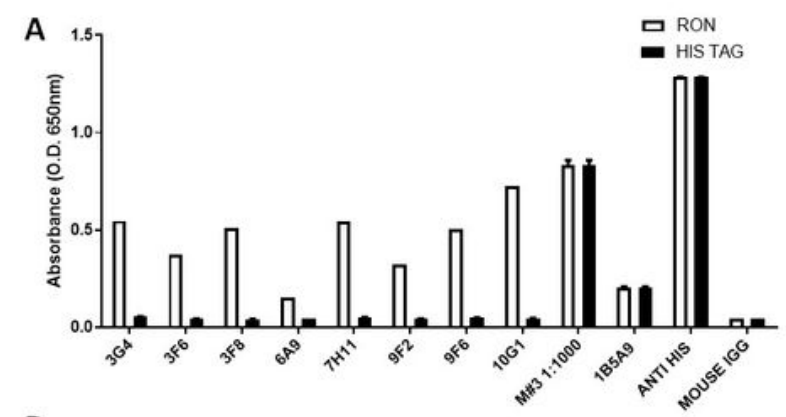

B
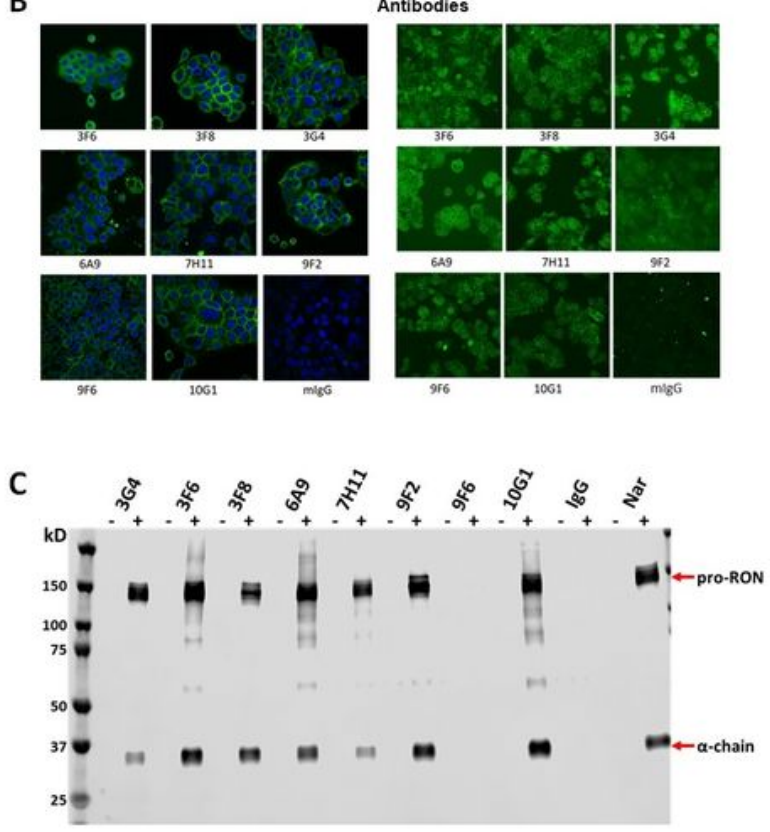

D

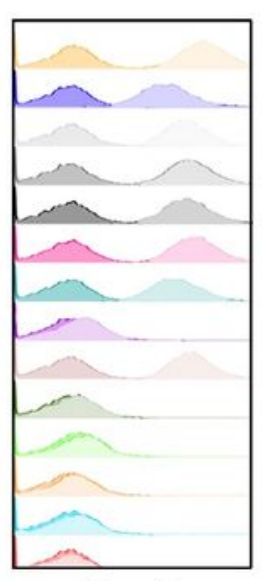

Live cells

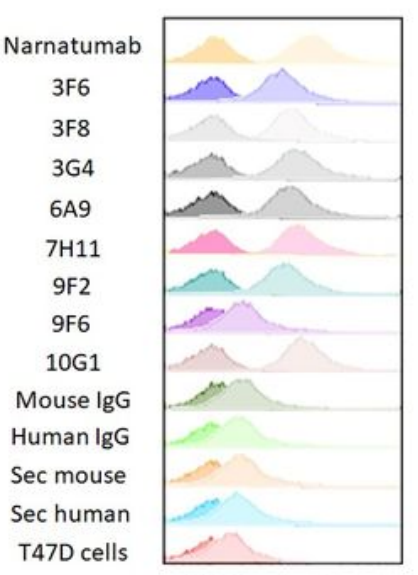

PFA fixed cells

\section{Figure 1}

Screening and characterization of RON antibodies via ELISA, cell staining, immunoprecipitation and flow cytometry A) A total of eight antibody clones were identified from the ELISA screen. Mammalian expressed extracellular RON antigen (His-tag) was coated on ELISA plates and His-tagged protein was used as a negative control. Immunized mouse serum (1:1000), 1B5A9 (commercial anti-RON antibody) and anti-His (commercial) was used as positive control antibodies and mlgG was used as a negative 
control. Absorbance was read at $650 \mathrm{~nm}$ using Envision plate reader (Perkin Elmer). B) Confocal images showing immunofluorescence staining of HCT116 cells and T47D cells using RON antibodies. Cells were stained with DAPI (blue) for nuclei visualisation and antibodies (green) using anti-mouse secondary antibody conjugated with Alexa Fluor 488. Polyclonal mouse IgG was used as a negative control. Scale bar: $50 \mu \mathrm{m} \mathrm{C)}$ Endogenous RON was immunoprecipitated using anti-RON antibodies. Narnatumab was used as a positive control and mouse IgG was used as a negative control. Eluted protein was resolved on an SDS page gel and homemade anti-alpha RON antibody 6 E6 was used to probe for immunoprecipitated RON. D) Anti-RON antibodies were used to stain live cells and fixed T47D cells (lighter peak) and T47D RON KO cells (darker peak) in flow cytometry. Anti-mouse secondary antibody conjugated with Alexa Fluor 488 (Invitrogen) was used for visualization of antibody staining. 


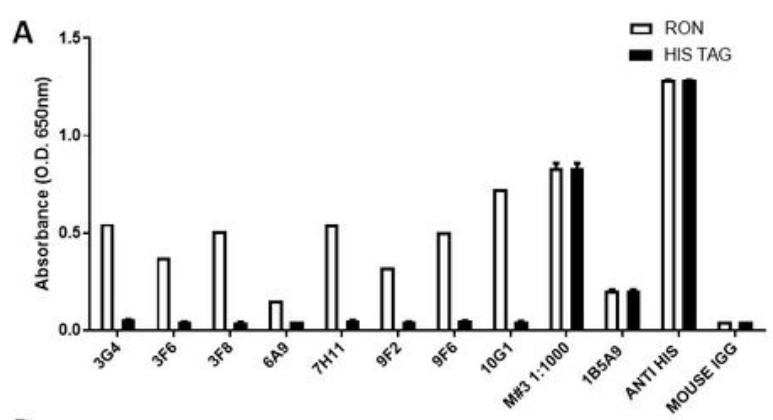

B
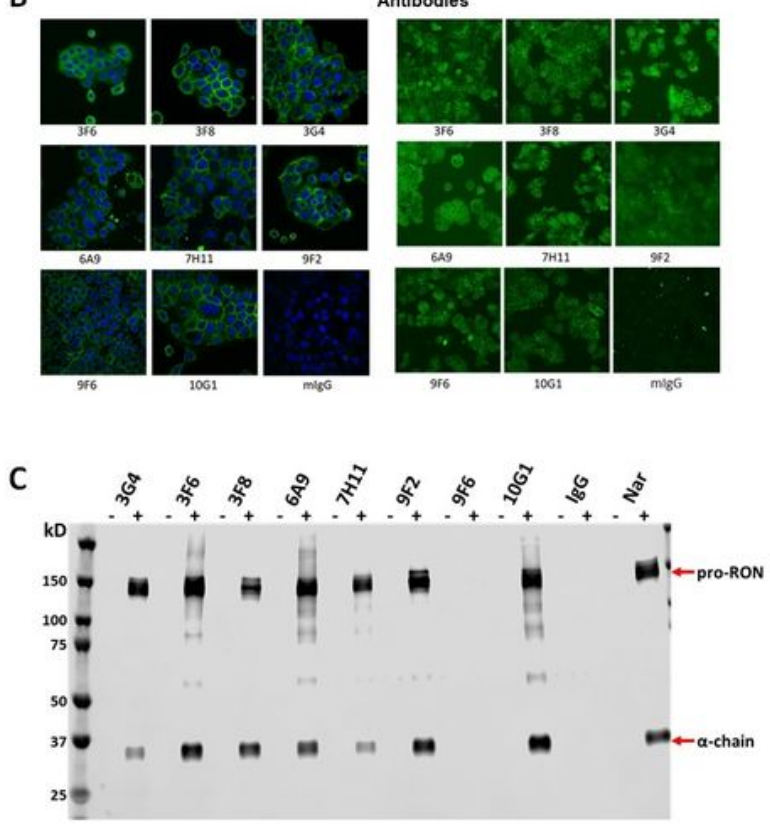

D

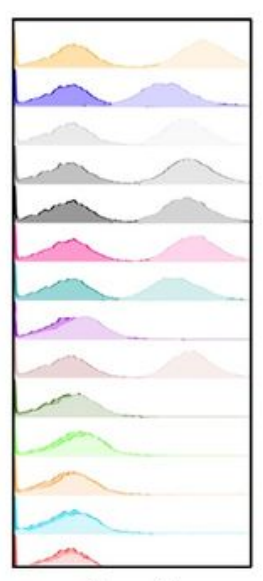

Live cells

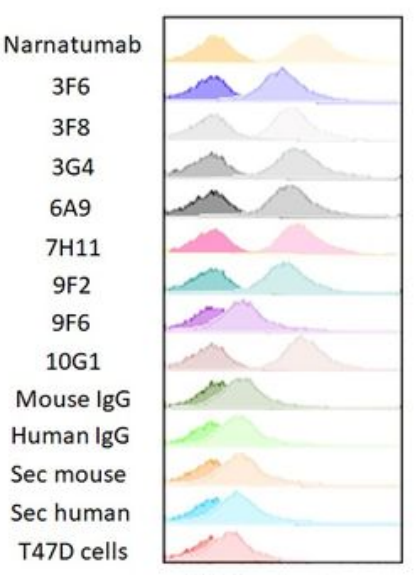

PFA fixed cells

\section{Figure 1}

Screening and characterization of RON antibodies via ELISA, cell staining, immunoprecipitation and flow cytometry A) A total of eight antibody clones were identified from the ELISA screen. Mammalian expressed extracellular RON antigen (His-tag) was coated on ELISA plates and His-tagged protein was used as a negative control. Immunized mouse serum (1:1000), 1B5A9 (commercial anti-RON antibody) and anti-His (commercial) was used as positive control antibodies and mlgG was used as a negative 
control. Absorbance was read at $650 \mathrm{~nm}$ using Envision plate reader (Perkin Elmer). B) Confocal images showing immunofluorescence staining of HCT116 cells and T47D cells using RON antibodies. Cells were stained with DAPI (blue) for nuclei visualisation and antibodies (green) using anti-mouse secondary antibody conjugated with Alexa Fluor 488. Polyclonal mouse IgG was used as a negative control. Scale bar: $50 \mu \mathrm{m} \mathrm{C)}$ Endogenous RON was immunoprecipitated using anti-RON antibodies. Narnatumab was used as a positive control and mouse IgG was used as a negative control. Eluted protein was resolved on an SDS page gel and homemade anti-alpha RON antibody 6 E6 was used to probe for immunoprecipitated RON. D) Anti-RON antibodies were used to stain live cells and fixed T47D cells (lighter peak) and T47D RON KO cells (darker peak) in flow cytometry. Anti-mouse secondary antibody conjugated with Alexa Fluor 488 (Invitrogen) was used for visualization of antibody staining. 

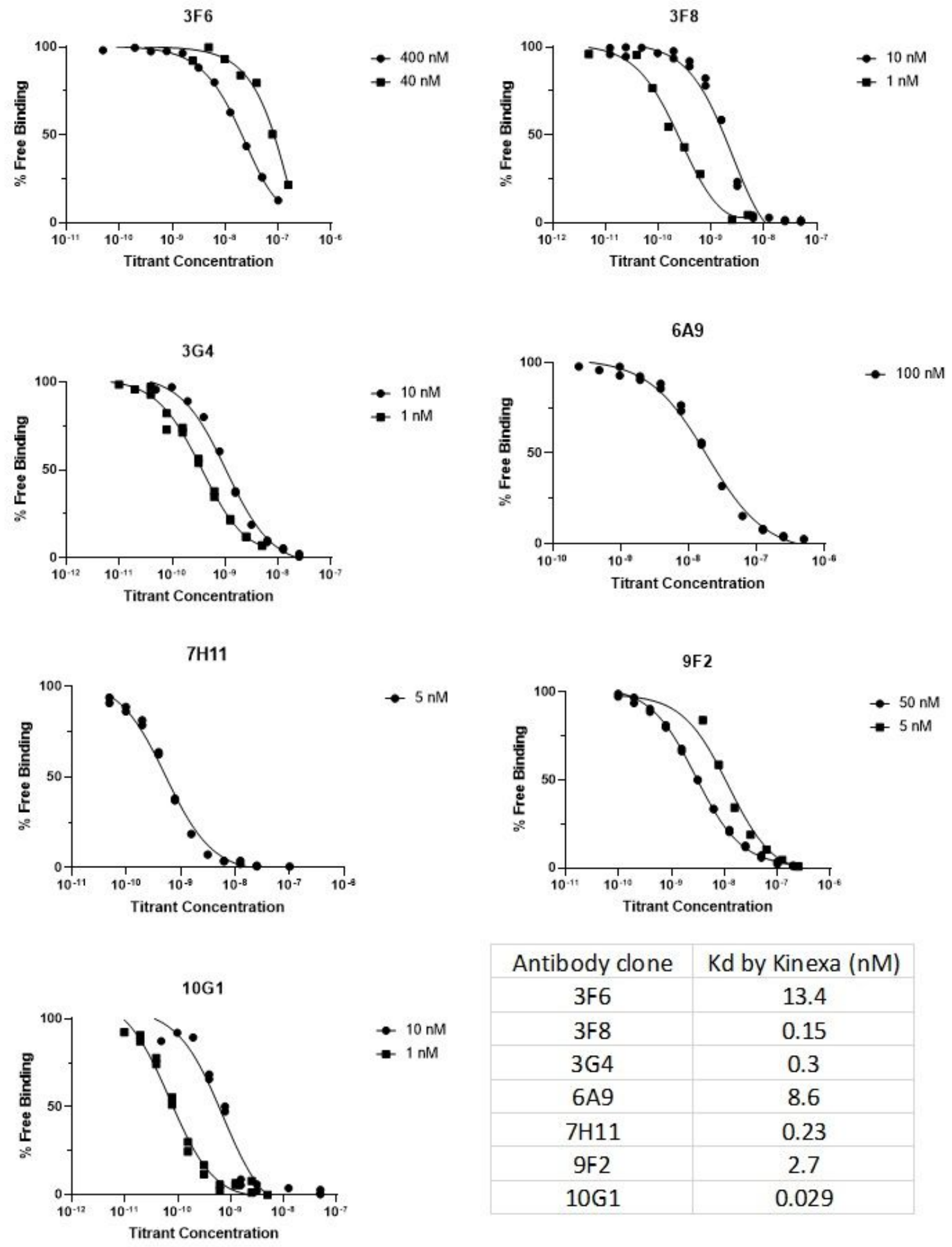

\begin{tabular}{|c|c|}
\hline Antibody clone & Kd by Kinexa $(\mathrm{nM})$ \\
\hline $3 \mathrm{~F} 6$ & 13.4 \\
\hline $3 \mathrm{~F} 8$ & 0.15 \\
\hline $3 \mathrm{G} 4$ & 0.3 \\
\hline $6 \mathrm{~A} 9$ & 8.6 \\
\hline 7H11 & 0.23 \\
\hline 9F2 & 2.7 \\
\hline 10G1 & 0.029 \\
\hline
\end{tabular}

\section{Figure 2}

KineXa determined binding affinities of anti-RON antibodies on mammalian expressed extracellular RON antigens Titration curves were generated using indicated fixed concentrations of extracellular RON antigens measured by calibration free concentration analysis (CFCA). Increasing concentrations of RON antibodies were titrated for measurement of binding. Apparent $\mathrm{Kd}$ values were determined from an average of the two curves and plotted in table shown. 

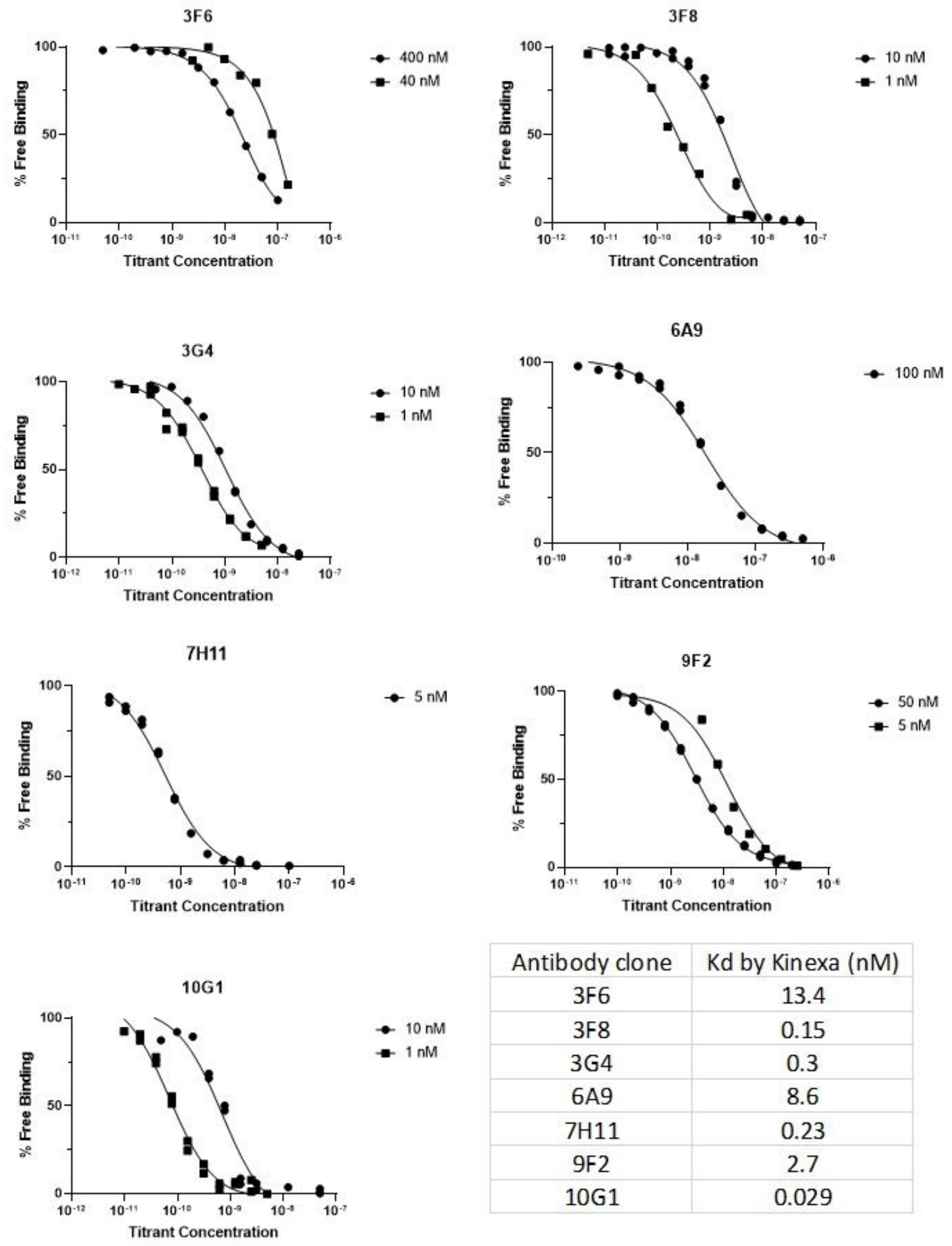

\begin{tabular}{|c|c|}
\hline Antibody clone & Kd by Kinexa $(\mathrm{nM})$ \\
\hline $3 F 6$ & 13.4 \\
\hline $3 F 8$ & 0.15 \\
\hline $3 G 4$ & 0.3 \\
\hline $6 A 9$ & 8.6 \\
\hline $7 H 11$ & 0.23 \\
\hline 9F2 & 2.7 \\
\hline $10 \mathrm{G} 1$ & 0.029 \\
\hline
\end{tabular}

\section{Figure 2}

KineXa determined binding affinities of anti-RON antibodies on mammalian expressed extracellular RON antigens Titration curves were generated using indicated fixed concentrations of extracellular RON antigens measured by calibration free concentration analysis (CFCA). Increasing concentrations of RON antibodies were titrated for measurement of binding. Apparent $\mathrm{Kd}$ values were determined from an average of the two curves and plotted in table shown. 
A

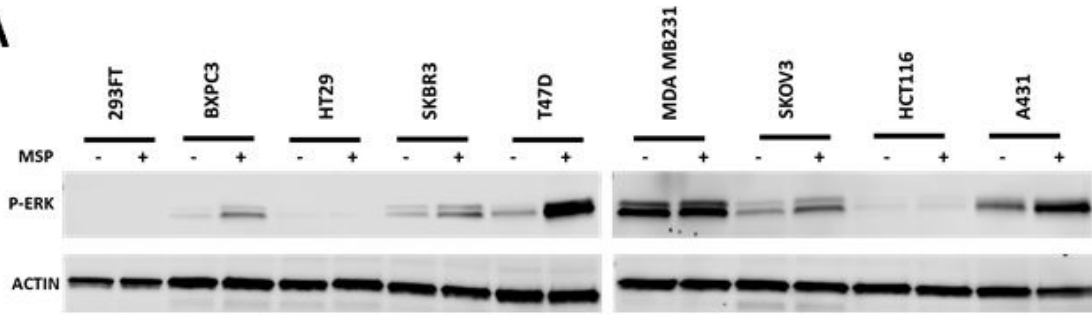

B

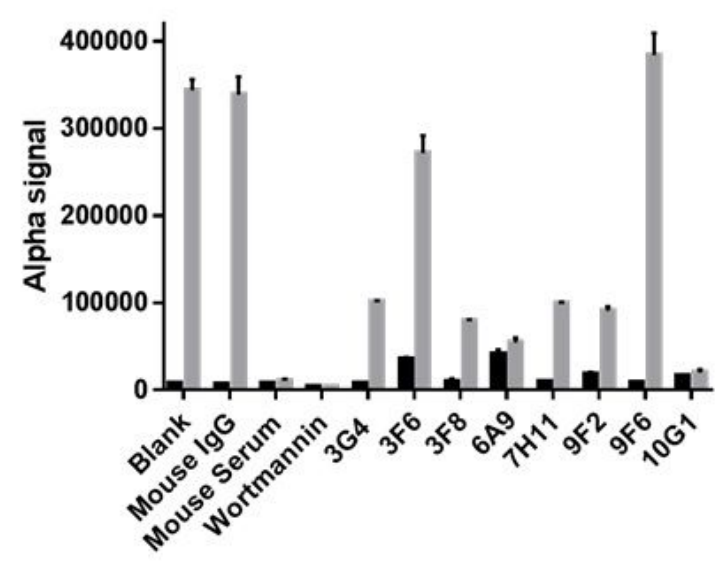

C

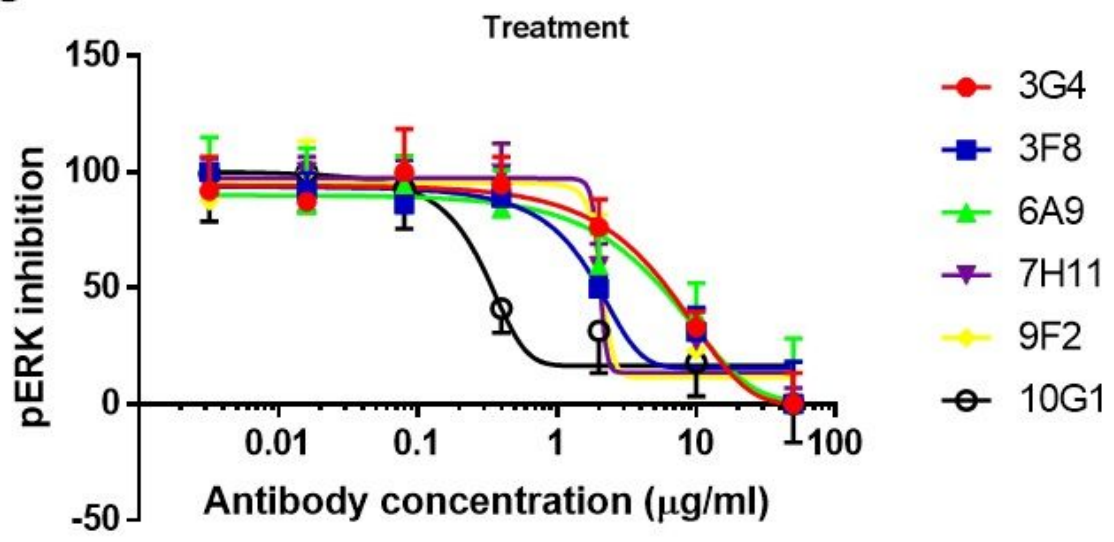

D

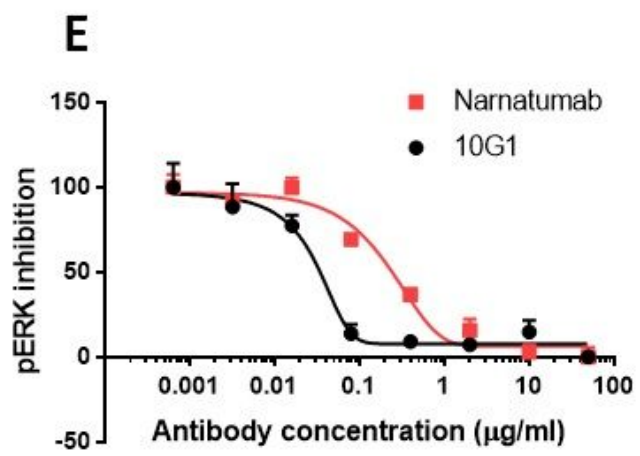

Figure 3

Anti-RON antibodies exerts antagonistic effects on MSP induced increase in levels of phosphorylated ERK A) RON expressing cell lines were probed for levels of phosphorylated ERK following stimulation by MSP. 293FT cell line was used as a negative control. Phospho-p44/42 MAPK (Erk1/2) (Thr202/Tyr204) Antibody \#9101(Cell signalling technologies) was used as a primary antibody. Secondary anti-rabbit-HRP (Jackson Laboratories) was used to visualize western binding. $\beta$-actin served as a loading control B) 
T47D cells and T47D RON knockout cells were treated with $10 \mu \mathrm{g} / \mathrm{ml}$ anti-RON antibodies for an hour followed by MSP for half an hour. Cells were harvested and probed for p-ERK levels with AlphaLISA surefire Ultra p-ERK Assay Kit (Perkin Elmer). C) T47D cells were treated with increasing concentrations (333 nM to $4 \mathrm{fM}$ ) anti-RON antibodies for an hour followed by MSP for half an hour. Cells were harvested and probed for p-ERK levels with AlphaLISA surefire Ultra p-ERK Assay Kit (Perkin Elmer). IC50 titration curves were plotted and calculated IC50 values were drawn in the table below. Wortmannin was used as a positive control and mouse IgG was used as negative control. D) EC50 values and ranking of the antibodies in PERK inhibition assays. E) T47D cells were treated with increasing concentrations of 10G1 and Narnatumab antibodies for an hour followed by MSP for half an hour. p-ERK levels were probed and EC50 values were calculated in the same ways as in Figure 3C. 
A

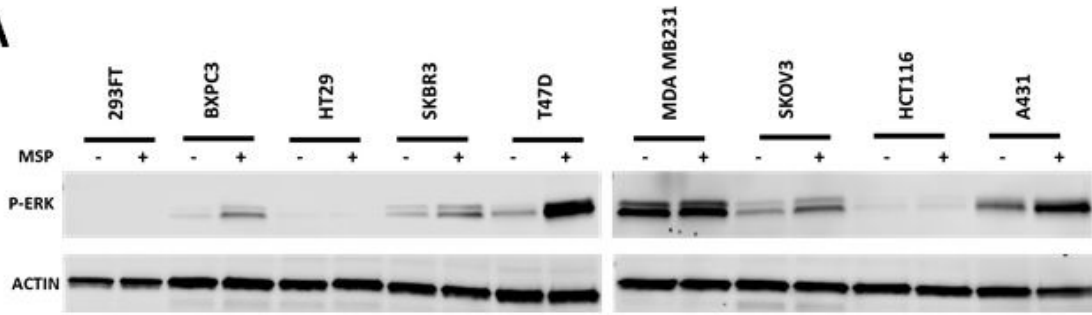

B

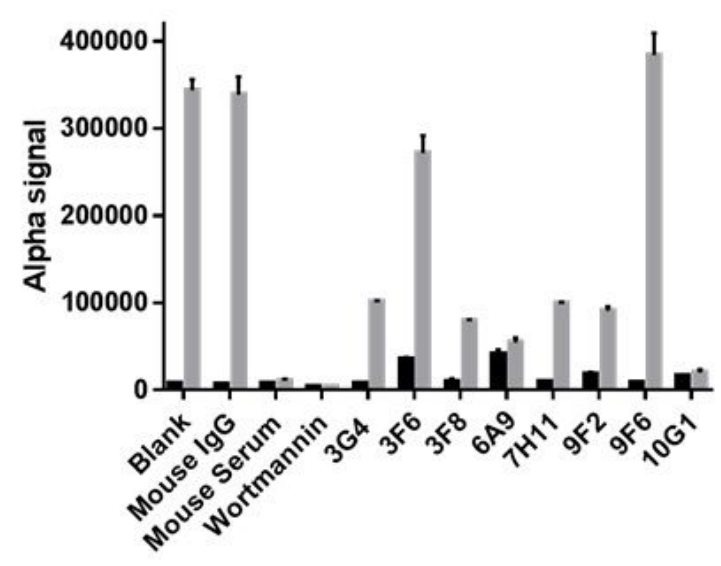

C

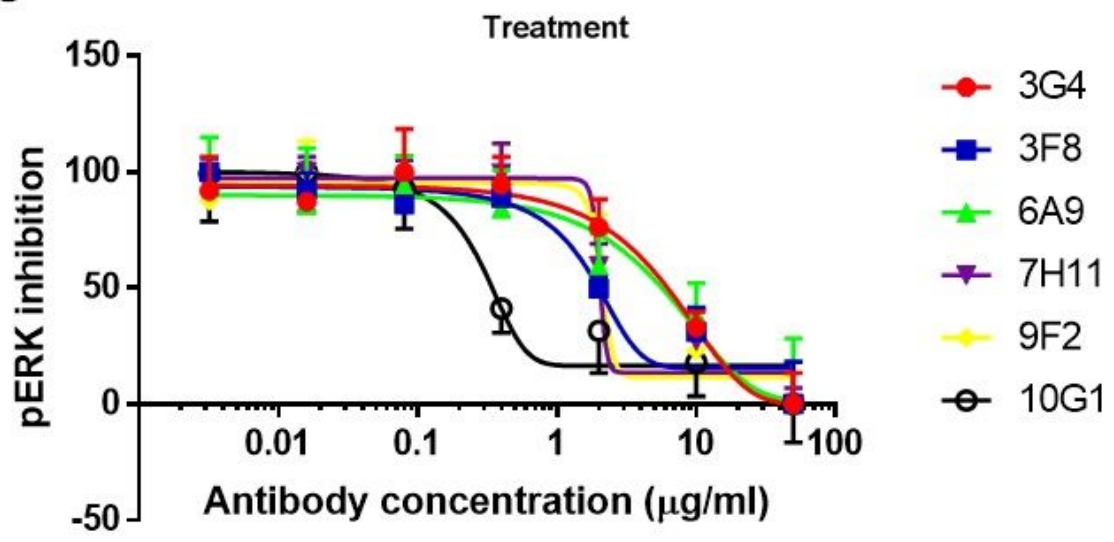

D

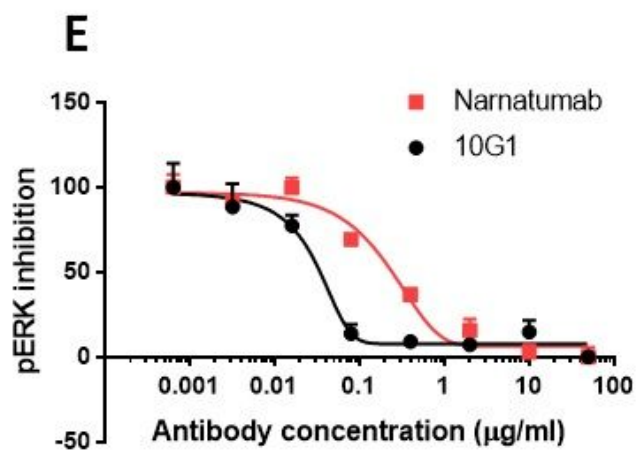

Figure 3

Anti-RON antibodies exerts antagonistic effects on MSP induced increase in levels of phosphorylated ERK A) RON expressing cell lines were probed for levels of phosphorylated ERK following stimulation by MSP. 293FT cell line was used as a negative control. Phospho-p44/42 MAPK (Erk1/2) (Thr202/Tyr204) Antibody \#9101(Cell signalling technologies) was used as a primary antibody. Secondary anti-rabbit-HRP (Jackson Laboratories) was used to visualize western binding. $\beta$-actin served as a loading control B) 
T47D cells and T47D RON knockout cells were treated with $10 \mu \mathrm{g} / \mathrm{ml}$ anti-RON antibodies for an hour followed by MSP for half an hour. Cells were harvested and probed for p-ERK levels with AlphaLISA surefire Ultra p-ERK Assay Kit (Perkin Elmer). C) T47D cells were treated with increasing concentrations (333 nM to $4 \mathrm{fM}$ ) anti-RON antibodies for an hour followed by MSP for half an hour. Cells were harvested and probed for p-ERK levels with AlphaLISA surefire Ultra p-ERK Assay Kit (Perkin Elmer). IC50 titration curves were plotted and calculated IC50 values were drawn in the table below. Wortmannin was used as a positive control and mouse IgG was used as negative control. D) EC50 values and ranking of the antibodies in PERK inhibition assays. E) T47D cells were treated with increasing concentrations of 10G1 and Narnatumab antibodies for an hour followed by MSP for half an hour. p-ERK levels were probed and EC50 values were calculated in the same ways as in Figure 3C. 
A
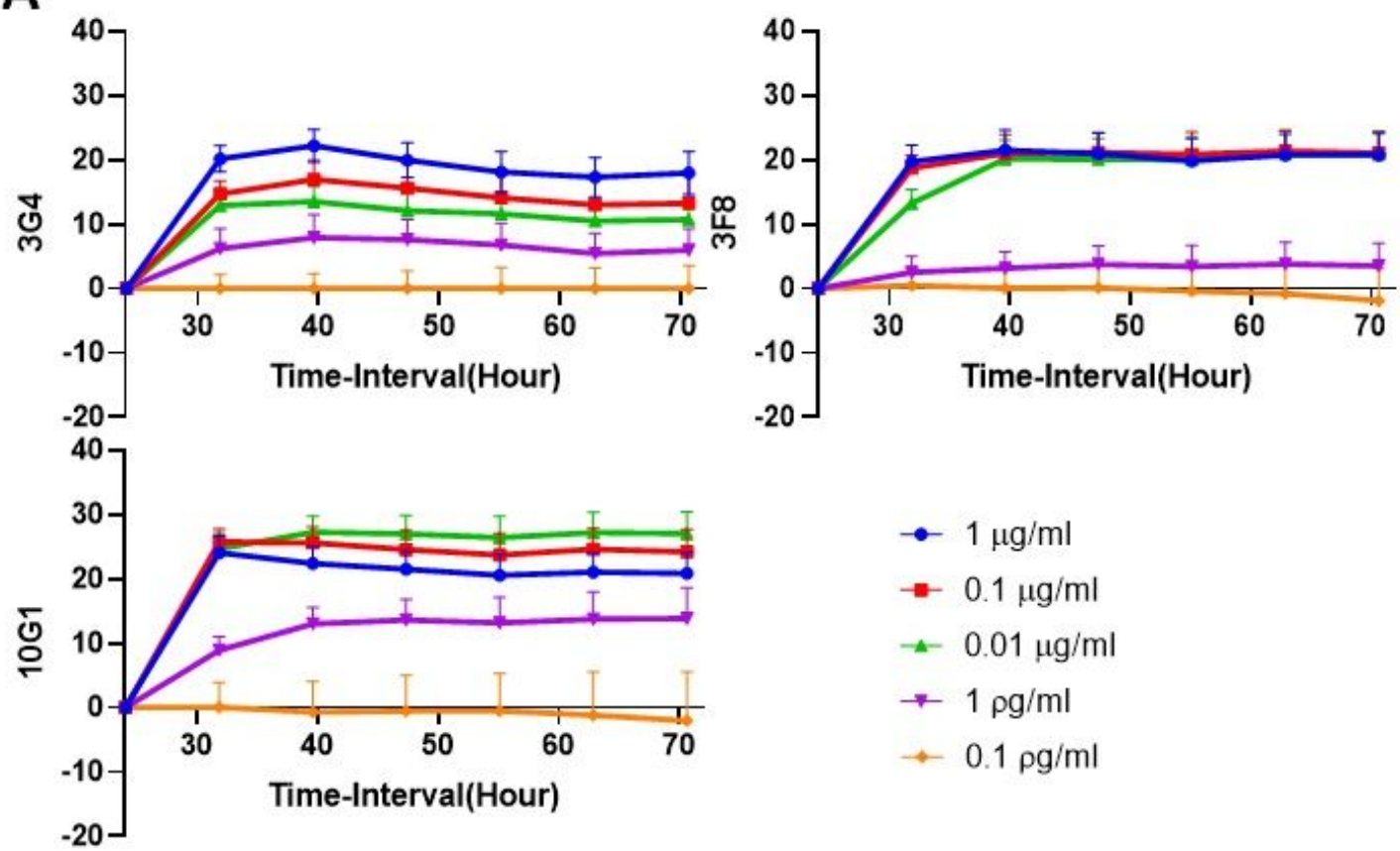

$$
\begin{aligned}
& \rightarrow 1 \mu \mathrm{g} / \mathrm{ml} \\
& -0.1 \mu \mathrm{g} / \mathrm{ml} \\
& \rightarrow 0.01 \mu \mathrm{g} / \mathrm{ml} \\
& \rightarrow 1 \rho \mathrm{gl} / \mathrm{ml} \\
& \rightarrow 0.1 \rho \mathrm{g} / \mathrm{ml}
\end{aligned}
$$

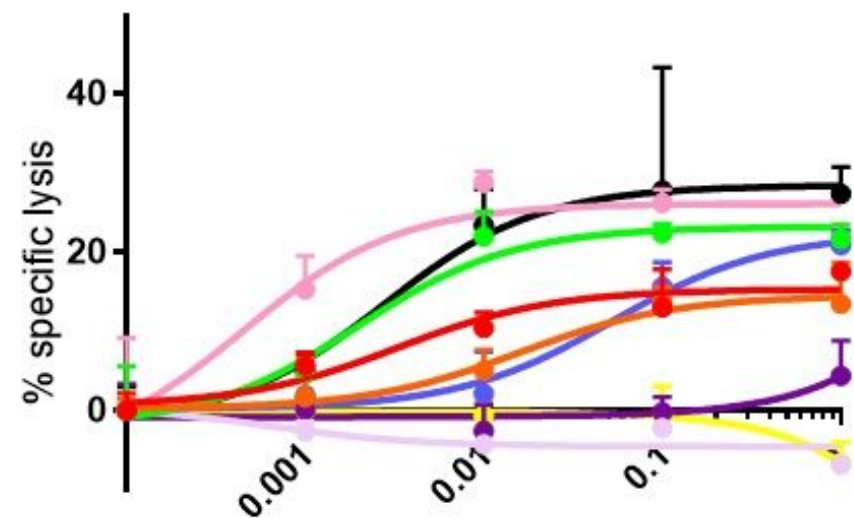

Antibody concentration $(\mu \mathrm{g} / \mathrm{ml})$

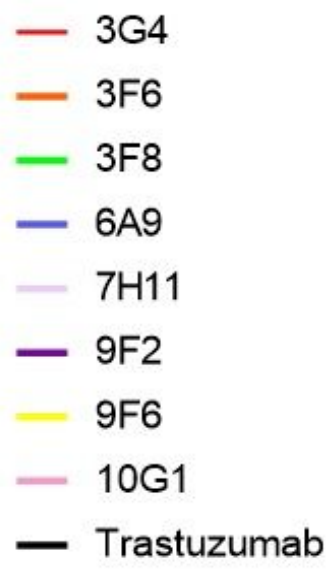

C

\begin{tabular}{|l|l|l|}
\hline Antibody & Ranking & EC50 (pM) \\
\hline 10G1 & 1 & 3.0 \\
\hline 3F8 & 2 & 13.0 \\
\hline 3G4 & 3 & 21.9 \\
\hline 3F6 & 4 & 107.6 \\
\hline 6A9 & 5 & 351.5 \\
\hline 7H11 & 6 & No activity \\
\hline 9F2 & 7 & No activity \\
\hline 9F6 & 8 & No activity \\
\hline
\end{tabular}

\section{Figure 4}

Antibody dependent cellular cytotoxicity activities of anti-RON antibodies tested in vitro A) All eight antiRON antibodies (chimeric mouse VH with human Fc) were tested for their abilities to induce antibody dependent cellular cytotoxicity (ADCC). Humanized Trastuzumab was used as a positive control. Target T47D cancer cells were seeded overnight for baseline measurement. Purified human NK were chosen as effector cells an E:T ratio of 10:1. Varying concentrations of antibodies $(0.1 \mathrm{pg} / \mathrm{ml}$ to $1 \mu \mathrm{g} / \mathrm{ml})$ were 
tested on target cells. Impedance based assay xCELLigence (Acea Bio) was used for quantification of ADCC activities. Percentage of cytolysis plot was calculated and plotted by $x C E L L i g e n c e ~ I m m u n o t h e r a p y$ software (Acea Bio). Data plotted starts from addition of effector cells and antibodies. Representative graphs of 3G4, 3F8 and 10G1 are shown. B) Normalized EC50 titration curve data of percentage cytolysis of individual antibodies and Trastuzumab at 24 hours following addition of effector cells and antibodies. EC50 values were noted and summarized in (C).

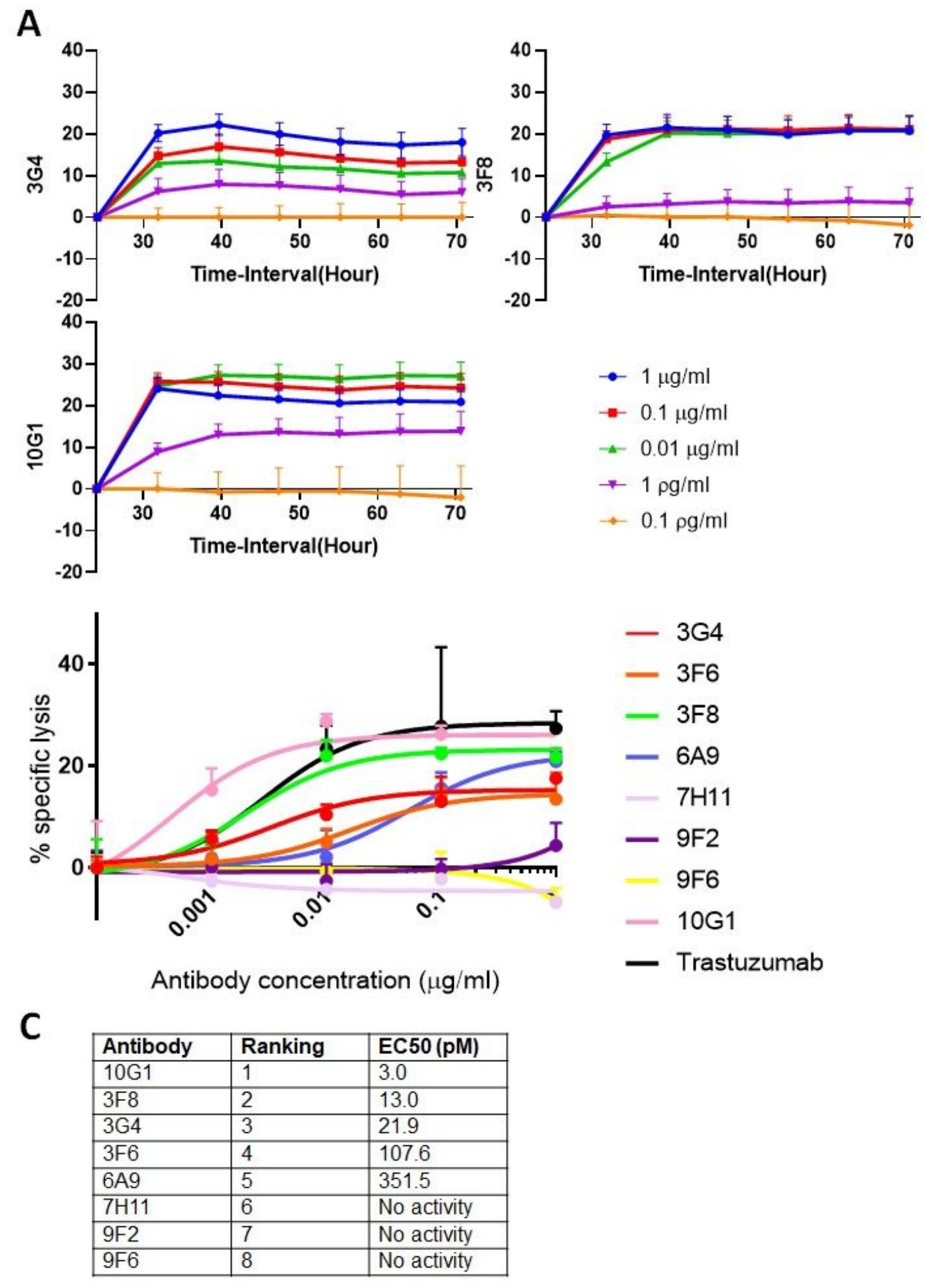

Figure 4 
Antibody dependent cellular cytotoxicity activities of anti-RON antibodies tested in vitro A) All eight antiRON antibodies (chimeric mouse VH with human Fc) were tested for their abilities to induce antibody dependent cellular cytotoxicity (ADCC). Humanized Trastuzumab was used as a positive control. Target T47D cancer cells were seeded overnight for baseline measurement. Purified human NK were chosen as effector cells an E:T ratio of 10:1. Varying concentrations of antibodies $(0.1 \mathrm{pg} / \mathrm{ml}$ to $1 \mu \mathrm{g} / \mathrm{ml})$ were tested on target cells. Impedance based assay xCELLigence (Acea Bio) was used for quantification of ADCC activities. Percentage of cytolysis plot was calculated and plotted by xCELLigence Immunotherapy software (Acea Bio). Data plotted starts from addition of effector cells and antibodies. Representative graphs of 3G4, 3F8 and 10G1 are shown. B) Normalized EC50 titration curve data of percentage cytolysis of individual antibodies and Trastuzumab at 24 hours following addition of effector cells and antibodies. EC50 values were noted and summarized in (C). 


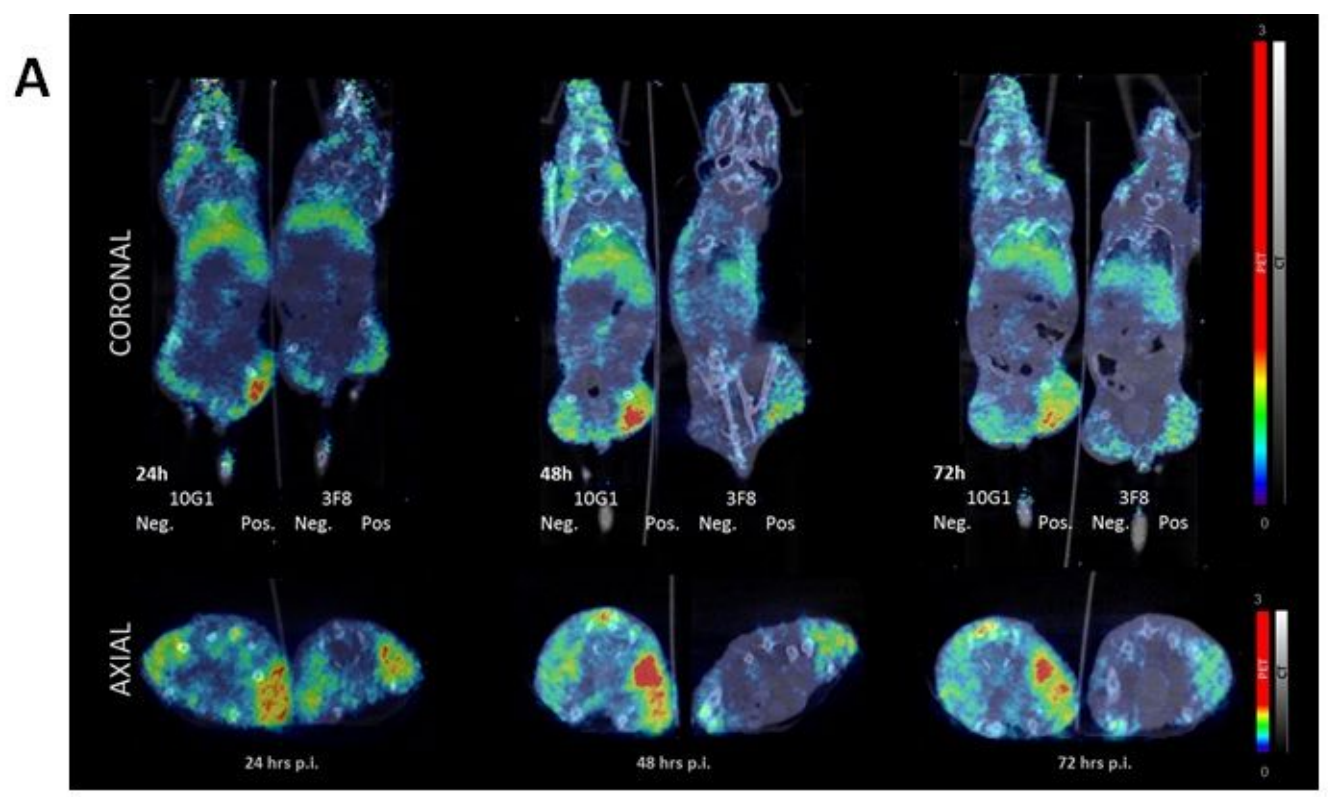

B

C
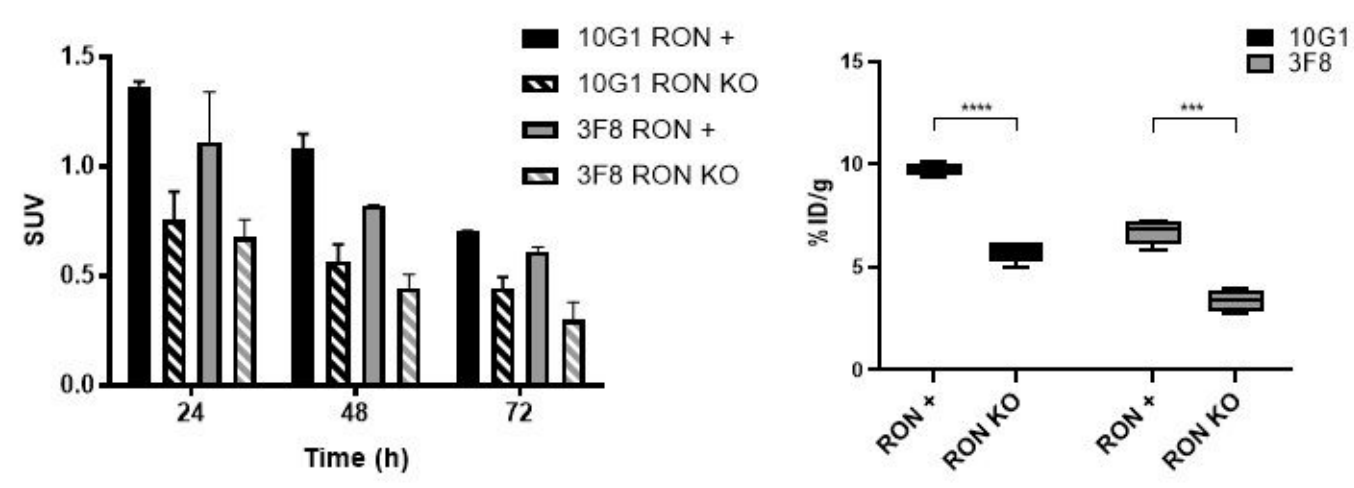

D

$E$
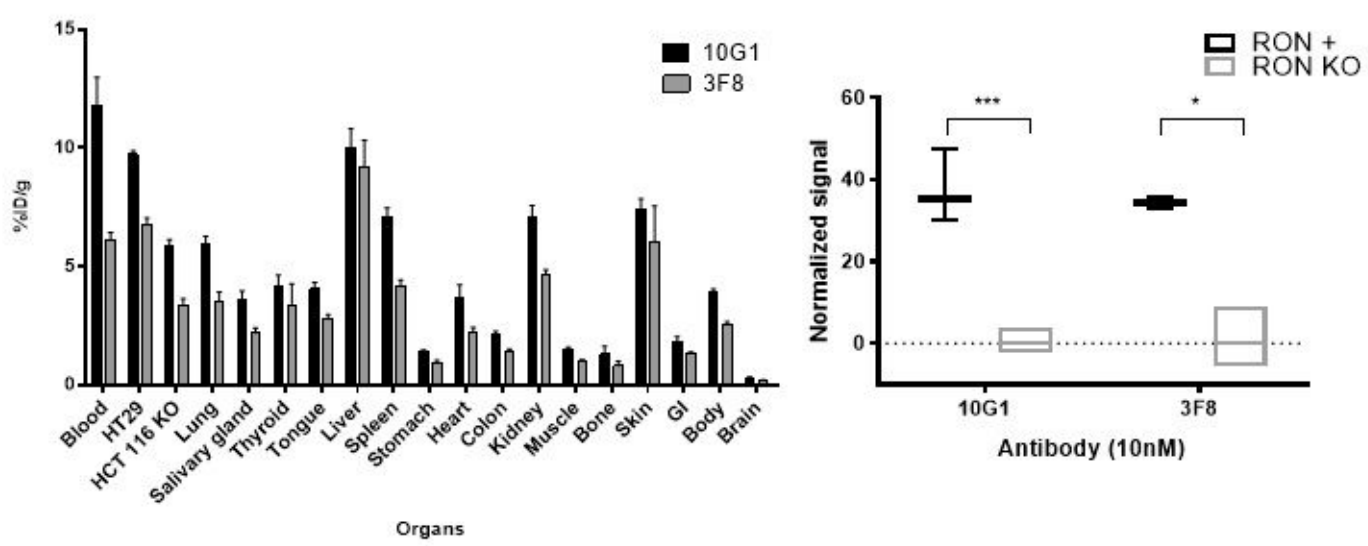

Figure 5

RON antibodies selectively targets RON-positive HT29 tumor xenografts A) Whole-body coronal and axial small-animal PET/CT images of RON positive HT29 (right flank) and RON negative HCT116 (left flank) tumor-bearing mice at 24,48 , and $72 \mathrm{~h}$ after intravenous injection of $89 \mathrm{Zr}$ - labelled $10 \mathrm{G} 1$ and 3F8. B) Standardized uptake (SUV) in RON positive and RON negative tumors of 89Zr-10G1 and 89Zr-3F8 over 72 $\mathrm{h}(\mathrm{n}=2$, error bars $=$ SD). C) Comparison of 89Zr-10G1 and 89Zr-3F8 uptake in RON positive and RON 
negative tumors, presented as percent injected dose per gram tumor $(n=4$, error bars=SD). D) 89Zr-10G1 and $89 \mathrm{Zr}-3 \mathrm{~F} 8$ uptake in tumors and major organs $72 \mathrm{~h}$ p.i., presented as percent injected dose per gram of tissue $(n=4$, error bars $=S D)$. E) In-vitro radioimmunoassay evaluating cellular binding of $89 \mathrm{Zr}-10 \mathrm{G} 1$ and $89 \mathrm{Zr}-3 F 8$ on the RON positive cell line in relation to the RON negative cell line $(n=2-3$, error bars $=$ $\mathrm{SD})$

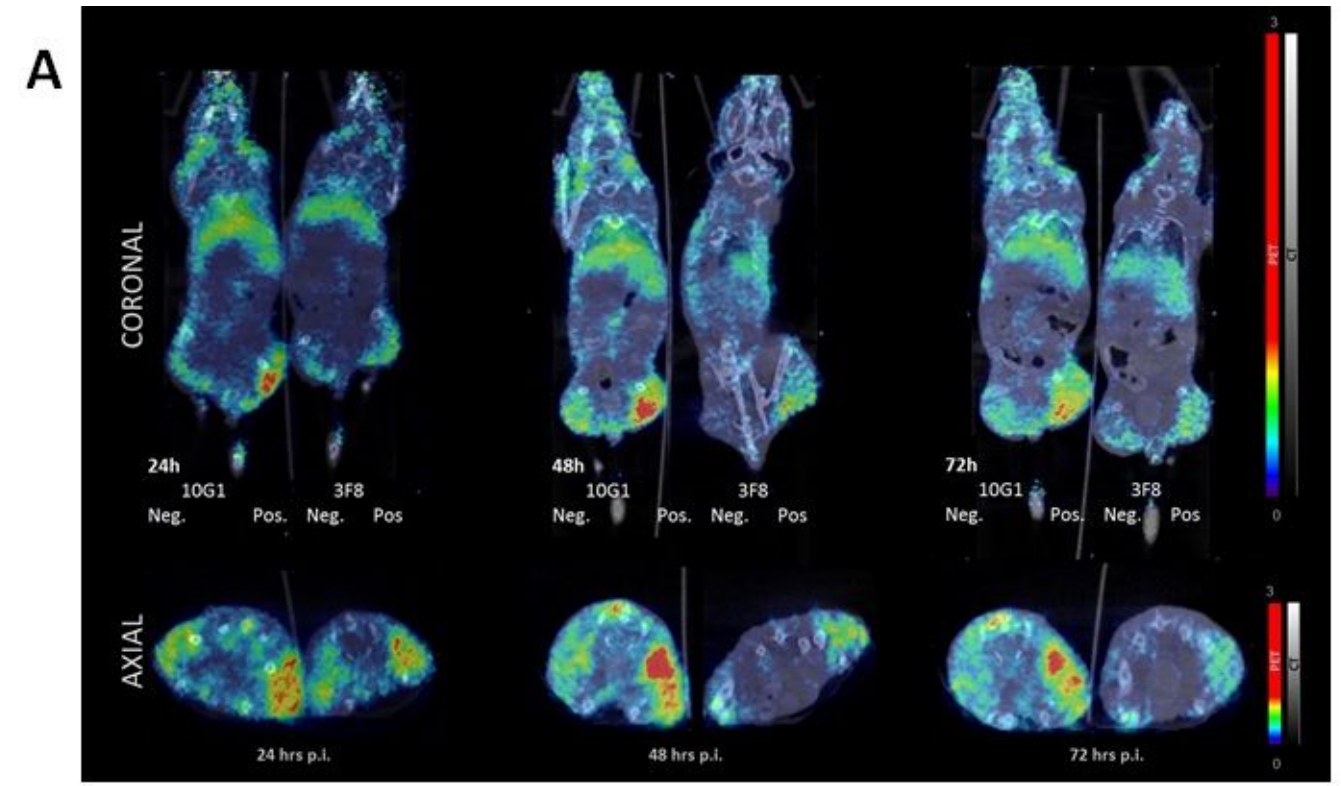

B

\section{C}

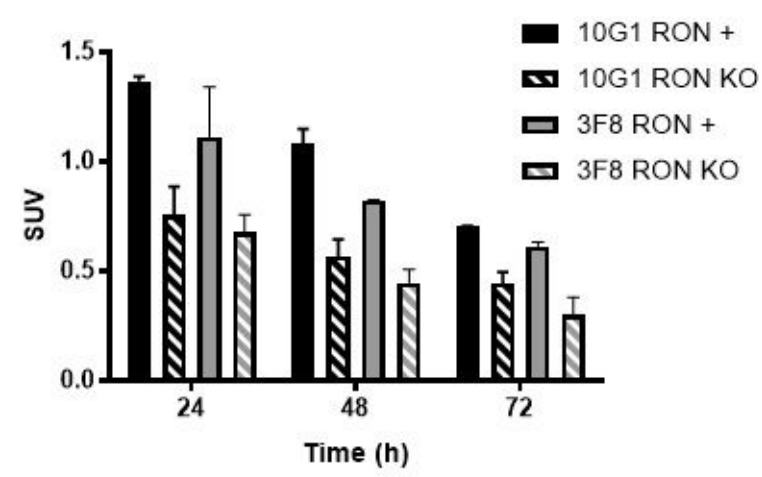

D
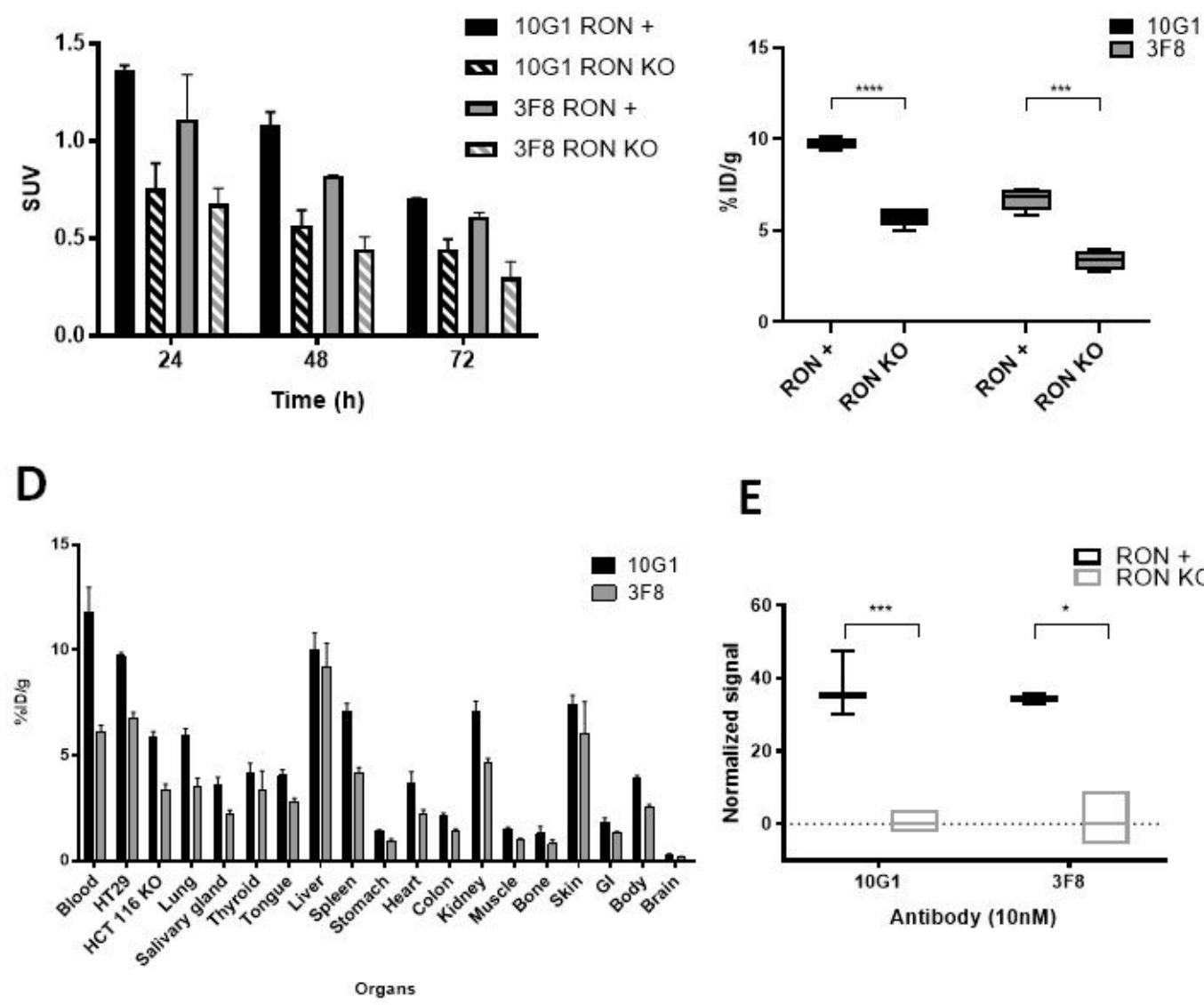

$E$

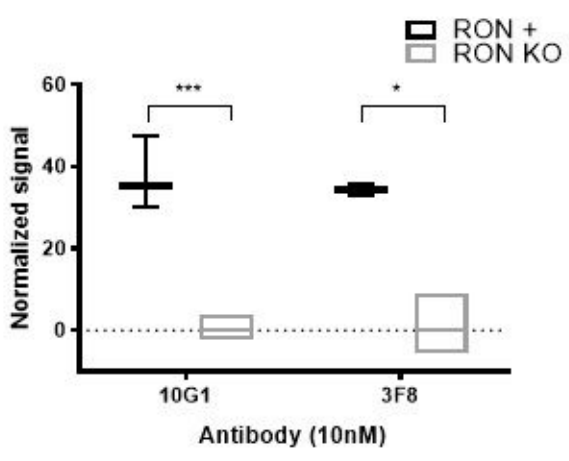

Figure 5 
RON antibodies selectively targets RON-positive HT29 tumor xenografts A) Whole-body coronal and axial small-animal PET/CT images of RON positive HT29 (right flank) and RON negative HCT116 (left flank) tumor-bearing mice at 24,48 , and $72 \mathrm{~h}$ after intravenous injection of $89 \mathrm{Zr}$ - labelled $10 \mathrm{G} 1$ and $3 \mathrm{~F} 8$. B) Standardized uptake (SUV) in RON positive and RON negative tumors of 89Zr-10G1 and 89Zr-3F8 over 72 $h(n=2$, error bars $=S D)$. C) Comparison of 89Zr-10G1 and 89Zr-3F8 uptake in RON positive and RON negative tumors, presented as percent injected dose per gram tumor $(n=4$, error bars=SD). D) 89Zr-10G1 and 89Zr-3F8 uptake in tumors and major organs $72 \mathrm{~h}$ p.i., presented as percent injected dose per gram of tissue $(n=4$, error bars = SD). E) In-vitro radioimmunoassay evaluating cellular binding of 89Zr-10G1 and $89 \mathrm{Zr}-3 \mathrm{~F} 8$ on the RON positive cell line in relation to the RON negative cell line ( $n=2-3$, error bars $=$ $\mathrm{SD})$ 